\title{
Stellar populations in NGC 5128 with the VLT: Evidence for recent star formation ${ }^{\star}$
}

\author{
M. Rejkuba ${ }^{1,2}$, D. Minniti ${ }^{2}$, D. R. Silva ${ }^{1}$, and T. R. Bedding ${ }^{3}$ \\ 1 European Southern Observatory, Karl-Schwartzschild-Strasse 2, 85748 Garching, Germany \\ e-mail: dsilva@eso.org \\ 2 Department of Astronomy, P. Universidad Católica, Casilla 306, Santiago 22, Chile \\ e-mail: dante@astro.puc.cl \\ 3 School of Physics, University of Sydney 2006, Australia \\ e-mail: bedding@physics.usyd.edu.au
}

Received 31 May 2001 / Accepted 13 September 2001

\begin{abstract}
We resolve stars of the nearest giant elliptical galaxy NGC 5128 using VLT with FORS1 and ISAAC. We construct deep $U, V$ and $K_{\mathrm{s}}$ color-magnitude and color-color diagrams in two different halo fields (in the halo and in the north-eastern diffuse shell). In the outer, shell field, at $\sim 14 \mathrm{kpc}$ from the center of the galaxy, there is a significant recent star formation with stars as young as $10 \mathrm{Myr}$, approximately aligned with the prominent radio and X-ray jet from the nucleus of the host AGN. Ionized gas filaments are evident in ultraviolet images near the area where neutral HI and CO molecular gas was previously observed. The underlying stellar population of the halo of the giant elliptical is predominantly old with a very broad metallicity distribution. The presence of an extended giant branch reaching $M_{\mathrm{bol}}=-5 \mathrm{mag}$ suggests the existence of a significant intermediate-age AGB population in the halo of this galaxy.
\end{abstract}

Key words. galaxies: elliptical and lenticular, $\mathrm{cD}$ - galaxies: stellar content - stars: fundamental parameters galaxies: individual: NGC 5128

\section{Introduction}

Galaxies show a wide range of star formation activity and a large range of metallicities and ages in their stellar populations. Understanding the physical nature and origins of their stars is fundamental to understanding the formation and evolution of galaxies. The current knowledge of star formation histories of galaxies along the Hubble sequence is summarized by Kennicutt (1998). This knowledge is mainly based on the integrated photometric and spectroscopic studies through the predictions of populations synthesis of the integrated light of star clusters (Bica 1988) and galaxies (Bruzual \& Charlot 1993; Maraston 1998). The predictions of population synthesis can now be tested directly not only for globular clusters (e.g. Vazdekis et al. 2001), but also for nearby galaxies.

Send offprint requests to: M. Rejkuba,

e-mail: mrejkuba@eso.org

* Based on observations collected at the European Southern Observatory, Paranal, Chile, within the Observing Programmes 63.N-0229 and 65.N-0164, and in part on observations collected by the NASA/ESA Hubble Space Telescope, which is operated by AURA, Inc., under NASA contract NAS5-26555.
Stellar evolution theory provides predictions of the features expected in color-magnitude diagrams (CMDs) for stellar populations with different ages and metallicities (Renzini \& Fusi Pecci 1988; Chiosi et al. 1992; Gallart 1998; Aparicio 1998; Tolstoy 1998). Coupled with the improvements in telescope sizes, detector sensitivity, field of view and spatial resolution, compared with those a decade ago, the direct observations of the stellar content of nearby galaxies is becoming one of the most active areas of extragalactic research.

In the Local Group, indicators of the old stellar populations such as old main sequence turn-offs or at least the horizontal branch magnitudes, are within reach of available instrumentation (e.g. Hurley-Keller et al. 1998; Gallart et al. 1999; Held et al. 2000; Rejkuba et al. 2000). The recent results on the studies of the Local Group galaxies have been summarized by Mateo (1998), van den Bergh (1999, 2000) and Grebel (2000).

The Local Group contains galaxies representative of almost all the classes, except the important giant elliptical class of galaxies. The closest giant elliptical is NGC 5128 , the dominant galaxy in the Centaurus group at distance of $3.6 \pm 0.3 \mathrm{Mpc}$ (Harris et al. 1999; Soria et al. 1996; Hui et al. 1995; Tonry \& Schechter 1990). It has been 
extensively studied over the last 50 years (for an exhaustive review see Israel 1998).

Its popularity is not only due to its brightness, but also to its many unusual features: (i) there is a prominent dust band containing young stars and HiI regions (Unger et al. 2000; Wild \& Eckart 2000; Graham 1979); (ii) there is an active nucleus with a radio and $\mathrm{X}$-ray jet, radio lobes and optical filaments (Cooper et al. 1965; Feigelson et al. 1981; Kraft et al. 2000; Schreier et al. 1981; Clarke et al. 1992; Blanco et al. 1975; Dufour \& van den Bergh 1978); (iii) Malin et al. (1983) discovered a large number of faint narrow shells of stars surrounding the galaxy; (iv) Schiminovich et al. (1994) detected $4 \times 10^{8} M_{\odot}$ of Hi gas associated with the stellar shells, but slightly displaced outside the shells; and (v) most recently, molecular $\mathrm{CO}$ gas has been found associated with the Hi gas and the stellar shells (Charmandaris et al. 2000). All of these are clear indications of a recent interaction with a gas-rich galaxy.

The high resolution and sensitivity of WFPC2 on the Hubble Space Telescope (HST) enabled the first studies of the resolved old stellar populations in the halo of NGC 5128 (Soria et al. 1996; Harris et al. 1999; Harris \& Harris 2000; Mould et al. 2000). NICMOS on HST was used to resolve the stars in the near IR (Marleau et al. 2000) in the same field as the optical study of Soria et al. (1996; $9 \mathrm{kpc}$ south of the center of the galaxy). In these two studies, a small intermediate-age population of $\sim 5$ Gyr has been found, comprising up to $\sim 10 \%$ of the total stellar population in the halo. On the other hand, there are almost no intermediate-age stars in the field further out in the halo, at $\sim 20 \mathrm{kpc}$ from the galaxy center (Harris et al. 1999) nor at $\sim 31 \mathrm{kpc}$ (Harris \& Harris 2000). The comparison of the two results may indicate the presence of a gradient in the stellar population in the halo, as suggested by Marleau et al. (2000). However, the small field of view of HST puts serious limitations to the conclusions in the cases where the strong gradients in galaxy populations exist (see, for example, the case of Local Group dwarf galaxies like Leo I (Gallart et al. 1999; Held et al. 2000) or WLM (Minniti \& Zijlstra 1996, 1997)).

We present here a wide wavelength range photometry of the resolved stellar populations in NGC 5128 obtained from the ground with Very Large Telescope (VLT) in $U, V$ and $K_{\mathrm{s}}$ band. The deep and high-resolution VLT imaging, coupled with the much larger field of view than HST, enables us to address the questions of the gradients in stellar populations in the halo of this giant elliptical galaxy. The proximity of NGC 5128 provides an unusual opportunity for a direct study of shell stars. We use infrared-optical colour-magnitude diagrams of the shell to study the ages and metallicities of the stars belonging to the cannibalized galaxy.

\section{The data}

The observations were carried out with the ESO Very Large Telescope (VLT) at Paranal Observatory, Chile.
They consist of optical (Bessel $U$ - and $V$-band) and nearIR $\left(K_{\mathrm{s}}\right.$-band) images. We observed two fields in the halo of NGC 5128. Field $1\left(\alpha_{2000}=13^{\mathrm{h}} 26^{\mathrm{m}} 23.5^{\mathrm{s}}, \delta_{2000}=\right.$ $-42^{\circ} 52^{\prime} 00^{\prime \prime}$; Fig. 1) was centered on the prominent N-E shell, $\sim 14^{\prime}$ away from the center of the galaxy. Field 2 $\left(\alpha_{2000}=13^{\mathrm{h}} 25^{\mathrm{m}} 26^{\mathrm{s}}, \delta_{2000}=-43^{\circ} 10^{\prime} 00^{\prime \prime} ;\right.$ Fig. 2$)$ was chosen to overlap with the HST field of Soria et al. (1996) and lies at a distance of $\sim 9^{\prime}$ from the center of the galaxy. The optical data were obtained on 1999 July 11 and 12, while the $K_{\mathrm{s}}$-band images cover several epochs and will be used to search for long-period variable stars. All of the observations were secured in the service mode. Calibration data, bias, dark and flat-field images as well as photometric standards from Landolt (in optical; 1992) and Persson et al. (IR; 1998) catalogues, were supplied by the ESO calibration plan. The observations are summarized in Table 1.

\subsection{Optical observations and data reduction}

Two 15-min exposures in both $U$ and $V$ were acquired for each of the two fields in NGC 5128 using FORS1 (FOcal Reducer and low dispersion Spectrograph) on VLT Unit Telescope 1 (Antu). The FORS1 detector is a $2048 \times 2048$ Tektronix CCD, thinned and anti-reflection coated. The pixel size is $24 \times 24 \mu \mathrm{m}$. The field of view is $6.8 \times 6.8$ and the scale is $0^{\prime \prime} 2 /$ pixel.

For service observations in direct-imaging mode, the FORS1 CCD is read out in four-port read-out mode. We used the ESO pipeline reductions which are based on MIDAS package specially developed to reduce images with 4 different amplifiers. With it the overscan for each amplifier was subtracted individually and after the subtraction of bias the images were corrected for the flat-field.

\subsection{Near-IR observations and data reduction}

For the near-IR $K_{\mathrm{s}}$-band observations we used ISAAC, also on Antu. In this wavelength domain $(0.9-2.5 \mu \mathrm{m})$ the detector is a $1024 \times 1024$ Hawaii Rockwell array. The field of view is $2.5 \times 2.5$ and the scale is $0 . .147 /$ pixel. A series of 10 -s exposures were taken at each epoch, usually in groups of six, with the number of repeats depending on weather conditions and any technical problems. The total exposure time for each epoch is given in Table 1.

An additional $K_{\mathrm{s}}$-band observation of Field 2, with a total on-target exposure time of $45 \mathrm{~min}$, was acquired with the SOFI instrument at the ESO 3.5-m New Technology Telescope (NTT) at La Silla Observatory, Chile. The field of view of SOFI is $4.94 \times 4.94$ and the scale is $0.2292 /$ pixel.

We also obtained observations with the NIC3 array on HST, using the F222M filter, which is similar to (although narrower than) the standard $K$ filter. The NIC3 field of view is $51^{\prime \prime} .2 \times 51^{\prime \prime} .2$, much smaller than the one of ISAAC.

The standard procedure in reducing IR data consists of (i) dark subtraction, (ii) flat-field correction, (iii) sky subtraction, (iv) registering and combining the images. We did not use the ESO pipeline reduction product. 


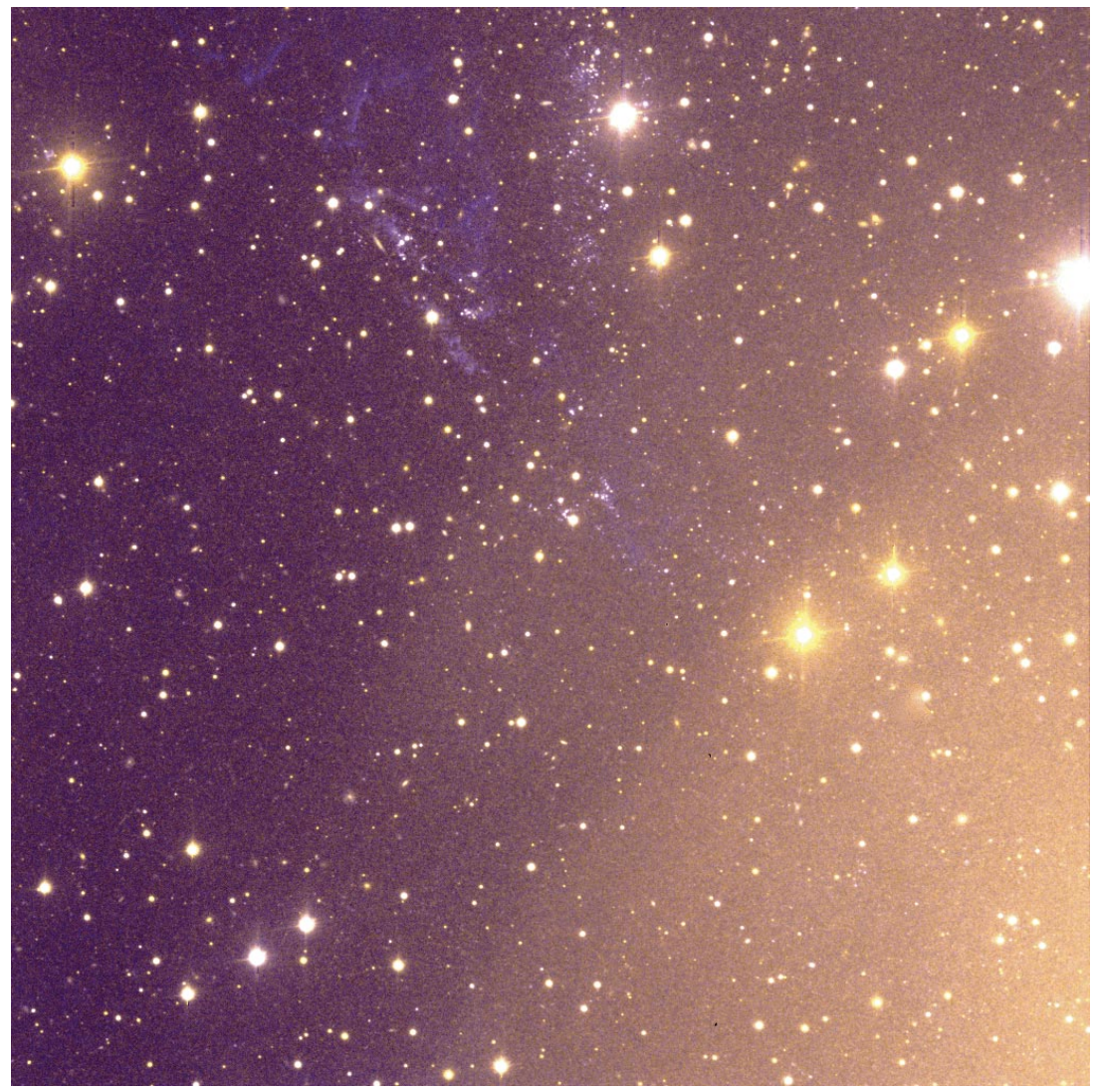

Fig. 1. Combined $U$ - and $V$-band image of Field 1 , taken at the VLT (UT1+FORS1). The field of view is $6.8 \times 6.8$. North is at the top and east to the left.

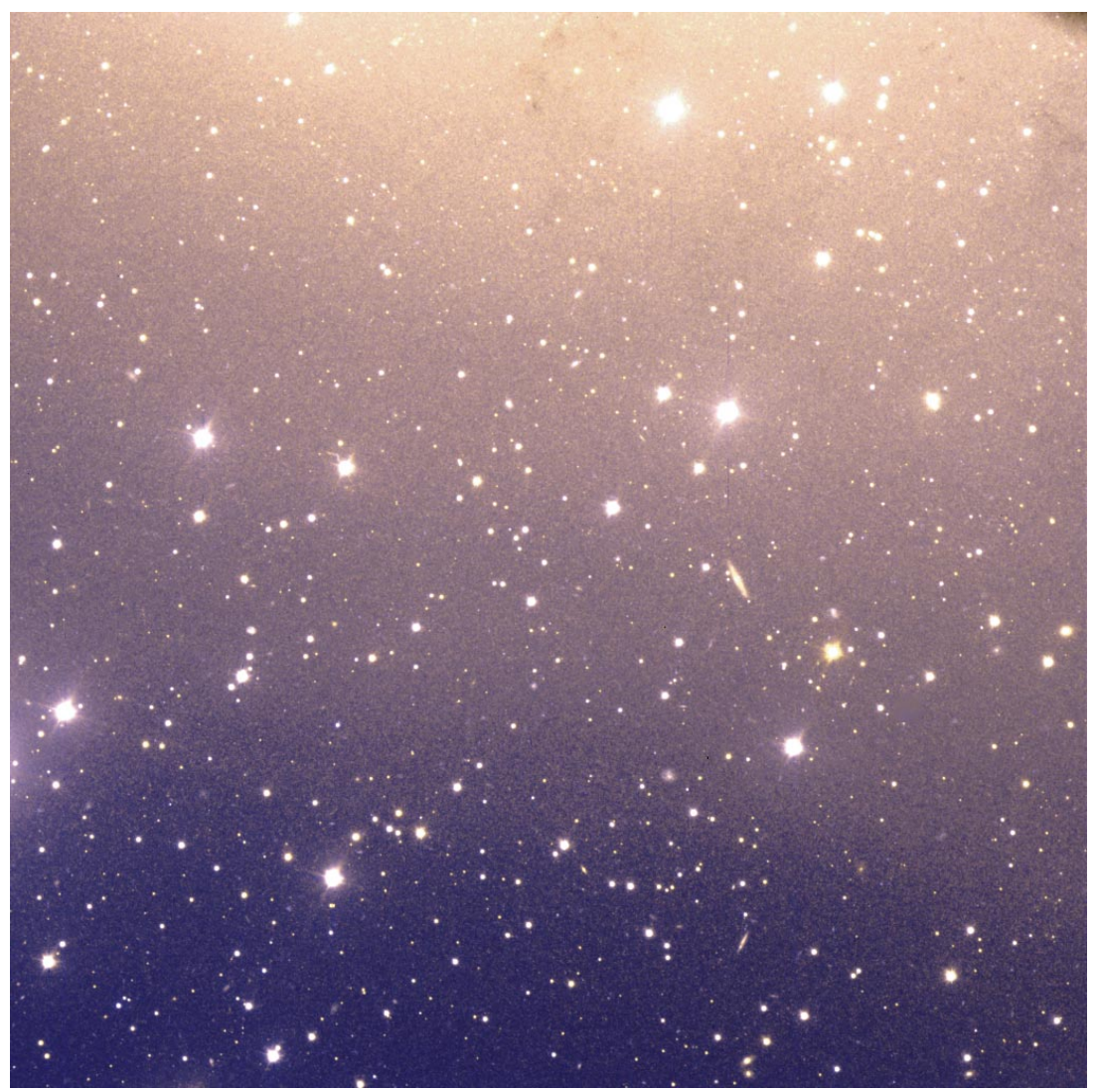

Fig. 2. Combined $U$ - and $V$-band image of Field 2, taken at the VLT (UT1+FORS1). The field of view is $6 !^{\prime} 8 \times 6{ }^{\prime} 8$. North is at the top and east to the left. 
Table 1. Journal of observations.

\begin{tabular}{|c|c|c|c|c|c|c|c|c|c|}
\hline $\begin{array}{l}\text { Field } \\
\# \\
\end{array}$ & $\begin{array}{l}\alpha_{(2000)} \\
(\mathrm{h} \text { min s) }\end{array}$ & $\begin{array}{l}\delta_{(2000)} \\
\left(\begin{array}{lll} & \prime & \prime \prime\end{array}\right) \\
\end{array}$ & $\begin{array}{l}\text { Date } \\
\text { dd/mm/yyyy }\end{array}$ & $\begin{array}{l}\text { Telescope \& } \\
\text { Instrument }\end{array}$ & $\begin{array}{l}\text { Exposure } \\
(\mathrm{s})\end{array}$ & Filter & Airmass & $\begin{array}{l}\text { Seeing } \\
\prime \prime\end{array}$ & Epoch \\
\hline 1 & 132623.5 & -425200 & $\overline{1} 12 / 07 / 1999$ & Antu+FORS1 & $2 \times 900$ & $\overline{\bar{U}}$ & 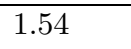 & 0.52 & \\
\hline 1 & 132623.5 & -425200 & 12/07/1999 & Antu+FORS1 & $2 \times 900$ & $V$ & 1.77 & 0.54 & \\
\hline 1 & 132623.5 & -425200 & 08/04/1999 & Antu+ISAAC & $10 \times 36 \times 10$ & $K_{\mathrm{s}}$ & 1.15 & 0.41 & A \\
\hline 1 & 132623.5 & -425200 & 07/05/1999 & Antu+ISAAC & $10 \times 36 \times 10$ & $K_{\mathrm{s}}$ & 1.05 & 0.40 & B \\
\hline 1 & 132623.5 & -425200 & 28/05/1999 & Antu+ISAAC & $10 \times 36 \times 10$ & $K_{\mathrm{s}}$ & 1.14 & 0.51 & $\mathrm{C}$ \\
\hline 1 & 132623.5 & -425200 & $15 / 04 / 2000$ & Antu+ISAAC & $65 \times 6 \times 10$ & $K_{\mathrm{s}}$ & 1.09 & 0.35 & $\mathrm{D}$ \\
\hline 1 & 132623.5 & -425200 & $10 / 05 / 2000$ & Antu+ISAAC & $65 \times 6 \times 10$ & $K_{\mathrm{s}}$ & 1.22 & 0.43 & $\mathrm{E}$ \\
\hline 1 & 132623.5 & -425200 & $07 / 06 / 2000$ & Antu+ISAAC & $65 \times 6 \times 10$ & $K_{\mathrm{s}}$ & 1.13 & 0.60 & $\mathrm{~F}$ \\
\hline 1 & 132623.5 & -425200 & $08 / 07 / 2000$ & Antu+ISAAC & $65 \times 6 \times 10$ & $K_{\mathrm{s}}$ & 1.15 & 0.31 & $\mathrm{G}$ \\
\hline 2 & 132524.0 & -431000 & 11/07/1999 & Antu+FORS1 & $2 \times 900$ & $U$ & 1.28 & 0.53 & \\
\hline 2 & 132524.0 & -431000 & 11/07/1999 & Antu+FORS1 & $2 \times 900$ & $V$ & 1.20 & 0.44 & \\
\hline 2 & 132524.0 & -431000 & 08/04/1999 & Antu+ISAAC & $10 \times 36 \times 10$ & $K_{\mathrm{s}}$ & 1.05 & 0.36 & A \\
\hline 2 & 132524.0 & -431000 & 07/05/1999 & Antu+ISAAC & $10 \times 36 \times 10$ & $K_{\mathrm{s}}$ & 1.10 & 0.39 & B \\
\hline 2 & 132524.0 & -431000 & 29/05/1999 & Antu+ISAAC & $10 \times 36 \times 10$ & $K_{\mathrm{s}}$ & 1.06 & 0.44 & $\mathrm{C}$ \\
\hline 2 & 132524.0 & -431000 & $15 / 04 / 2000$ & Antu+ISAAC & $65 \times 6 \times 10$ & $K_{\mathrm{s}}$ & 1.09 & 0.40 & $\mathrm{D}$ \\
\hline 2 & 132524.0 & -431000 & $12 / 05 / 2000$ & Antu+ISAAC & $51 \times 6 \times 10$ & $K_{\mathrm{s}}$ & 1.22 & 0.51 & $\mathrm{~Eb}$ \\
\hline 2 & 132524.0 & -431000 & $19 / 05 / 2000$ & Antu+ISAAC & $20 \times 6 \times 10$ & $K_{\mathrm{s}}$ & 1.22 & 0.44 & Ec \\
\hline 2 & 132524.0 & -431000 & $07 / 06 / 2000$ & Antu+ISAAC & $41 \times 6 \times 10$ & $K_{\mathrm{s}}$ & 1.13 & 0.57 & $\mathrm{Fa}$ \\
\hline 2 & 132524.0 & -431000 & $09 / 06 / 2000$ & Antu+ISAAC & $30 \times 6 \times 10$ & $K_{\mathrm{s}}$ & 1.13 & 0.40 & $\mathrm{Fb}$ \\
\hline 2 & 132524.0 & -431000 & $08 / 07 / 2000$ & Antu+ISAAC & $65 \times 6 \times 10$ & $K_{\mathrm{s}}$ & 1.15 & 0.40 & G \\
\hline 2 & 132524.0 & -430904 & $20 / 02 / 2000$ & NTT+SOFI & $45 \times 10 \times 6$ & $K_{\mathrm{s}}$ & 1.04 & 0.55 & \\
\hline 2 & 132524.3 & -430958 & $27 / 06 / 1998$ & $\mathrm{HST}+\mathrm{NIC} 3$ & 256 & $\mathrm{~F} 222 \mathrm{M}$ & & & \\
\hline
\end{tabular}

Good sky subtraction in a crowded field like that of a galactic halo is particularly important. For that step we used the DIMSUM package (Stanford et al. 1995) within $\mathrm{IRAF}^{1}$. In DIMSUM the sky subtraction is made in two passes. In the first, a median sky is computed for each image from the six frames that are closest in time. The shifts between the sky-subtracted frames are then computed and all the images stacked together using a rejection algorithm to remove cosmic rays. An object mask is computed for the coadded image and then shifted back in order to create object masks for the individual frames. In the second pass, the sky subtraction is made using the object masks to avoid overestimation of the sky level. These masks are also used to check that the bright object cores were not removed as cosmic rays in the previous pass. After the mask-pass sky subtraction, all frames belonging to a single epoch are registered with imalign and combined with imcombine task in IRAF.

\section{The photometry}

\subsection{Data analysis}

The photometric reduction of the combined $U-, V$ - and $K_{\mathrm{s}}$-band images was performed using the DAOPHOT II programme (Stetson 1987, 1994). First, we located all the objects that were $>3 \sigma$ above the background on individual

\footnotetext{
${ }^{1}$ IRAF is distributed by the National Optical Astronomy Observatories, which is operated by the Association of Universities for Research in Astronomy, Inc., under contract with the National Science Foundation.
}

images. More than 50 relatively bright, not saturated, isolated, stellar objects were chosen to create the variable PSF for each image. ALLSTAR fitting of the PSF to all the objects produced the object lists that were matched with DAOMATCH and DAOMASTER, where only objects with good photometry in at least two frames were kept. The final photometric catalogue was obtained with ALLFRAME which uses as input information photometry lists from ALLSTAR and fits the PSF to all frames (in $U$, $V$ and $K$-band) simultaneously. Again, only the objects detected in at least 2 images were kept. Using the information on the location of stars in all bands simultaneously improved our photometry, which is deeper by $\sim 1$ mag with respect to ALLSTAR photometry. Moreover, the treatment of close companions, in particular the ones that have different colors, is much better with ALLFRAME.

NGC 5128 is close enough that its globular clusters appear slightly resolved, in the sense of having a larger FWHM and non-stellar PSF (Minniti et al. 1996; Rejkuba 2001). Restricting the sharpness and goodness of the fit parameters to $-0.7<\mathrm{SHARP}<0.7$, we rejected most of the galaxies, star clusters and other extended objects as well as remaining blemishes and cosmic rays from the final photometry list. Also stars with large photometric uncertainties in one or more filters $(\sigma \geq 0.5 \mathrm{mag})$ were rejected. Finally the images were visually checked and a few remaining extended background objects (e.g. partially resolved star forming region in a background spiral galaxy) were discarded. With this selection our final $U, V$ photometry catalogue contains 1581 and 1944 stars in Fields 1 and 2, respectively, while the $V, K$ catalogue contains 5172 and 

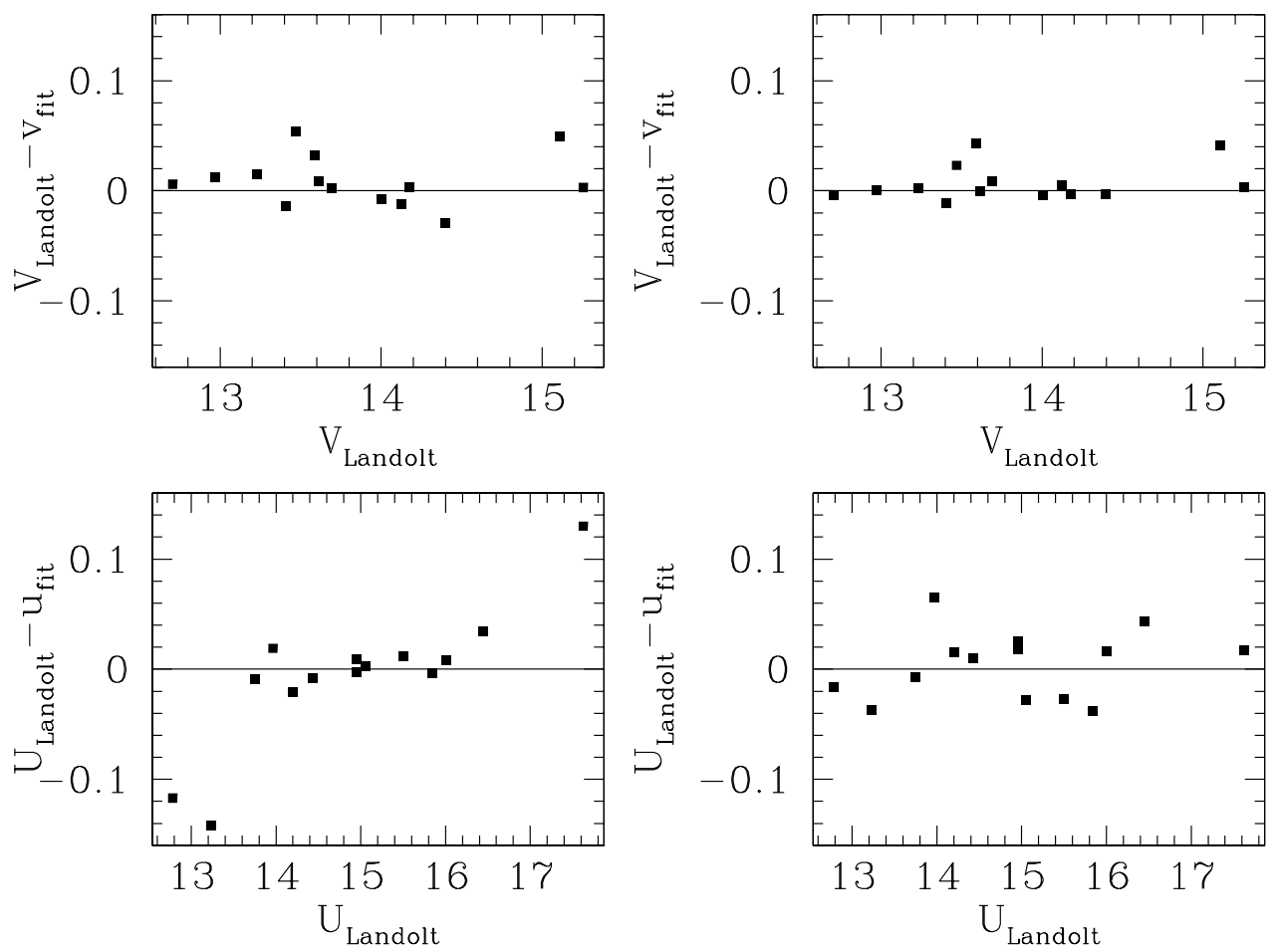

Fig. 3. Photometric calibration of Landolt (1992) stars during the nights of observations in $U$ and $V$ filters. Left panels: the scatter for the calibration without the color term; right panels: the scatter for the calibration with the color term.

8005 stars and the numbers of stars with good photometry in $U, V$ and $K$-band are 508 and 663 in Fields 1 and 2, respectively.

\subsection{The photometric calibration}

For the photometric calibration of the optical images, standard stars from the catalogue of Landolt (1992) were used. We checked the photometric quality of the two nights separately and since both were photometric, with almost identical zeropoints, we combined the standard stars for the two nights. Using a total of 14 stars in 4 different fields, spanning the color range $-1.321<(U-V)<$ 4.162 , we derived the following calibration transformations:

$$
\begin{aligned}
u_{\text {inst }}= & U-24.262( \pm 0.089)+0.379( \pm 0.068) * X \\
& -0.042( \pm 0.007) *(U-V) \\
v_{\text {inst }}= & V-27.348( \pm 0.042)+0.213( \pm 0.034) * X
\end{aligned}
$$

where $X$ is the mean airmass of the observations, $u_{\text {inst }}$ and $v_{\text {inst }}$ are instrumental magnitudes and $U$ and $V$ magnitudes from the Landolt (1992) catalogue. The one-sigma scatter around the mean was 0.031 mag for the $U$ band and $0.023 \mathrm{mag}$ for the $V$ band (Fig. 3, left panel). Adding the color term $(U-V)$ in the transformations slightly reduces the scatter in the $V$-band to $0.017 \mathrm{mag}$ (Fig. 3, right panel). However, the calibration equation without the color term for the $V$-band was preferred, since the scientific data in that filter go much deeper and $U$ magnitudes for some objects could not be measured accurately enough.
Observations at our reference $K_{\mathrm{s}}$-band epoch ( $\mathrm{G}$ in Table 1) were taken in photometric conditions. During the same night, July 8 2000, three standard stars from the list of Persson et al. (1998) were observed. Each standard star was observed at 5 different positions on the IR-array. In this way, a total of 15 independent measurements were obtained. However, the number of measurements with different airmass was only 3 , so that we preferred to adopt the mean extinction coefficients measured on Paranal for $K_{\mathrm{s}}$-band of $0.05 \mathrm{mag} /$ airmass. The derived zeropoint of the G-epoch observations is $24.23 \pm 0.04$. The following calibration equation was applied to our data:

$k_{\mathrm{s}, \text { inst }}=K_{\mathrm{s}}-24.23( \pm 0.04)+0.05 * X$

where $X$ is the airmass of observations, $k_{\mathrm{s}, \text { inst }}$ is the instrumental magnitude and $K_{\mathrm{s}}$ is the calibrated magnitude. The photometry of all other $K$-band epochs were measured with respect to the reference epoch.

\subsection{Completeness and contamination}

We made extensive tests to measure completeness and magnitude uncertainties as a function of magnitude and radial distance from the center of the galaxy. The completeness for the $U, V$ and $K_{\mathrm{s}}$-band photometry has been calculated using the ADDSTAR programme within DAOPHOT. We made twenty artificial star experiments, adding each time $\sim 3000$ stars to the first frame. The stars were added on a regular grid separated by $2 \times R_{P S F}+$ 1 pixels in order not to alter crowding (where $R_{P S F}$ is the PSF radius used for fitting the image with the worst 

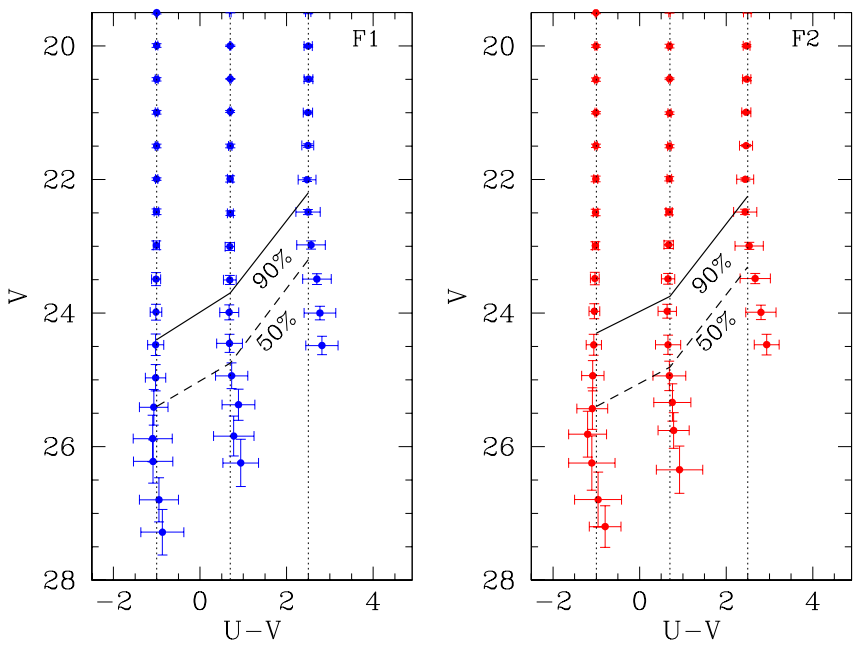

Fig. 4. The completeness and photometric uncertainties as a function of magnitude and color. The thin vertical dotted lines indicate the value of input $U-V$ colors. The full line defines the $90 \%$ completeness limit and the dashed line is for $50 \%$ completeness limit.
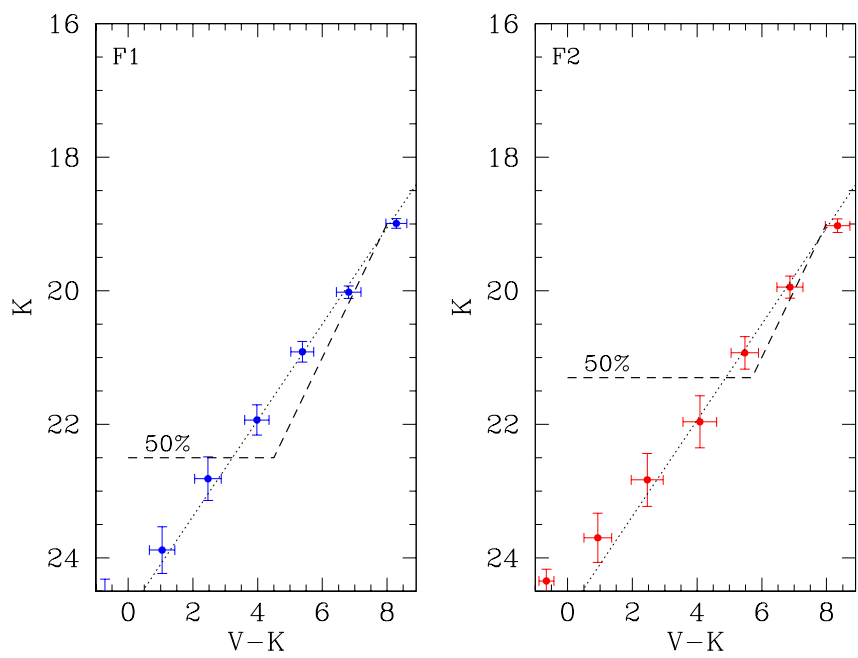

Fig. 5. The completeness and photometric uncertainties as a function of magnitude and color. The thin dotted line indicate the value of input $V-K$ colors. The dashed line defines the $50 \%$ completeness limit.

seeing) and having magnitudes randomly distributed in the observed range. The position of the first star in the list was chosen randomly, so that over 20 different experiments the added stars were uniformly distributed over the whole field. After the appropriate coordinate shifts were applied and magnitudes changed to the instrumental system taking into account the observed magnitudes and colors, the same stars from the first frame were added also to all other frames. Their photometry was recomputed in the same way as for the original images. The stellar PSF obtained from the field stars for the respective image was used in the simulations. Incompleteness in Field 1, defined by a recovery rate of $50 \%$ from the artificial-star experiment, sets in around magnitude 25 in the $U$-band (but depends strongly on $U-V$ color; see Fig. 4) and 22.5 in

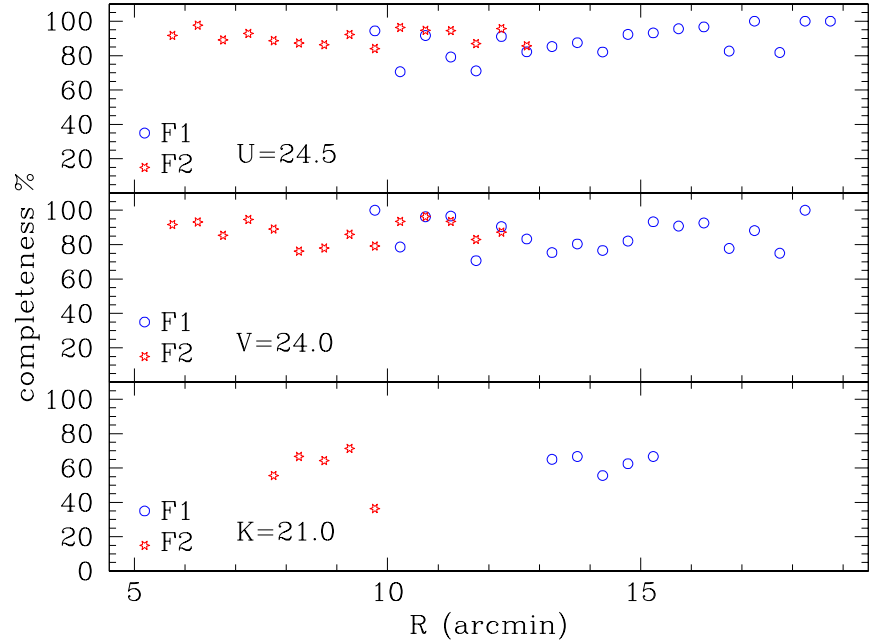

Fig. 6. The completeness as function of radial distance from the center of NGC 5128 calculated around 90\% completeness limit for $U$ - and $V$-band (for $(U-V)=0.7)$ and around $65 \%$ for $K_{\mathrm{s}}$-band.

$K_{\mathrm{s}}$-band (Fig. 5). The corresponding numbers for Field 2 are 25 for $U$-band and 21.3 for $K_{\mathrm{s}}$-band. The difference at $K_{\mathrm{s}}$-band between the two fields is due to (1) better seeing in Field $1(F W H M=2.1$ pix vs. 2.7 pix $)$ and (2) higher surface brightness in Field 2.

Dependence of the completeness on radial distance from the center of the galaxy $\left(\alpha_{2000}=13^{\mathrm{h}} 25^{\mathrm{m}} 26^{\mathrm{s}} 4\right.$, $\left.\delta_{2000}=-43^{\circ} 01^{\prime} 05^{\prime \prime} 1\right)$ was calculated for the magnitude bin around $90 \%$ level of completeness in $U$ - and $V$-band (Fig. 6) and around $65 \%$ of completeness in $K_{\mathrm{s}}$-band. There is no significant spatial variation of completeness in our data.

To assess the accuracy of our photometry, we calculated the difference between the input and recovered magnitudes for each magnitude bin (Figs. 4, 5). Our photometry is reliable down to the incompleteness limit and blending does not seriously affect our data (see the discussion in Sect. 4.2). For magnitudes fainter than the 50\% completeness limit, the measured values are systematically brighter, because of the bias towards brighter fluctuations and blending due to crowding. The colors of the recovered stars with magnitudes fainter than the $50 \%$ completeness limits are redder than the input colors in the $U V$ CMDs (Fig. 4), due to the larger incompleteness in the $U$ - than in the $V$-band. In the $V K$ CMDs the colors of the recovered stars range from slightly redder than the input color for the very red stars, due to the dominant incompleteness in the $V$-band, to bluer for the faintest and bluest stars, due to the dominant incompleteness in the $K$-band (Fig. 5). We did not correct our data for this systematic shift, because magnitudes fainter than the $50 \%$ completeness limit will not be used in further analysis.

Contamination by foreground Galactic stars and unresolved background galaxies is important because of the low Galactic latitude of our fields $(b=19.5)$ and the very deep photometric limits observed. We used the Besançon 
group model of stellar population synthesis of the Galaxy available through the $\mathrm{Web}^{2}$ (Robin \& Creze 1986; Robin et al. 1996) to simulate the total number and optical magnitude and color distribution of Galactic foreground stars in our fields. The simulated catalogue has 1827 stars in the FORS1 field $(6 ! 8 \times 6 ! 8)$ in the magnitude interval $18<V<30$. In order to get a realistic estimate for the number of stars that would be observed in our fields, the correction for completeness is necessary. Therefore, we added the stars from the simulated catalogue (with magnitudes scaled to correspond to instrumental magnitudes) to our images and re-measured their magnitudes. In this way, realistic photometric uncertainties were applied and the number of stars recovered in two fields was corrected for completeness. A total of 340 and 350 stars with $U$ and $V$-band photometry satisfying profile fitting and photometric uncertainty selection criteria were measured in Field 1 and 2, respectively. Most of the foreground stars have $0<U-V<3$ (Fig. 7). All of the stars in the red part of the Field $2 \mathrm{CMD}$ brighter than $V \sim 22$ are expected to be foreground stars (see Sect. 4.1). In order to adjust for the expected number of Galactic stars we normalized the models to the observed number of reddest stars in Field 2. Thus the total number of foreground stars was increased by $31 \%$ in both fields.

The Galaxy model simulation supplies not only the colors of the simulated stars, but also their metallicities, ages, spectral types and luminosity classes. Using all these data and the Kurucz (1998) model atmospheres ${ }^{3}$, we derived the foreground contamination in the $K$-band. After correction for completeness, the expected number of foreground stars in ISAAC images is 112 and 91 in the $V K$ CMD of Field 1 and 2, respectively. All of the foreground stars have $1.0<V-K<4.5$ (Fig. 7 right panel).

The measured number of compact background galaxies on the FORS1 images, taken in similar observing conditions, is $\$ 400$ in the magnitude range $V=20-25$ mag for the selection of sharpness parameter $-1<\operatorname{sharp}<1$ (Jerjen \& Rejkuba 2001). Our tighter selection criteria $(-0.7<$ sharp $<0.7)$ eliminated most of them. In the smaller field of view of ISAAC, the predicted number of background galaxies is $\sim 330$ in the interval of magnitudes $16<K_{\mathrm{s}}<23$ and $\sim 180$ between $16<K_{\mathrm{s}}<22$ (Saracco et al. 2001). Most of the background galaxies are resolved and rejected by sharp and magnitude uncertainty parameter requirements on our photometry. Only few compact galaxies might contaminate the sample.

\subsection{Comparison with published data}

Several recent studies of resolved stellar populations in NGC 5128 exist in the literature, all but one made with HST in F606W $(V)$ and F814W $(I)$ or F110W $(J)$ and F160W $(H)$ photometric bands, which are not very sensitive to the recent star formation. Our Field 2 is centered

\footnotetext{
2 http://www.obs-besancon.fr/www/modele/modele_ang.html

3 http://cfaku5.harvard.edu/grids.html
}
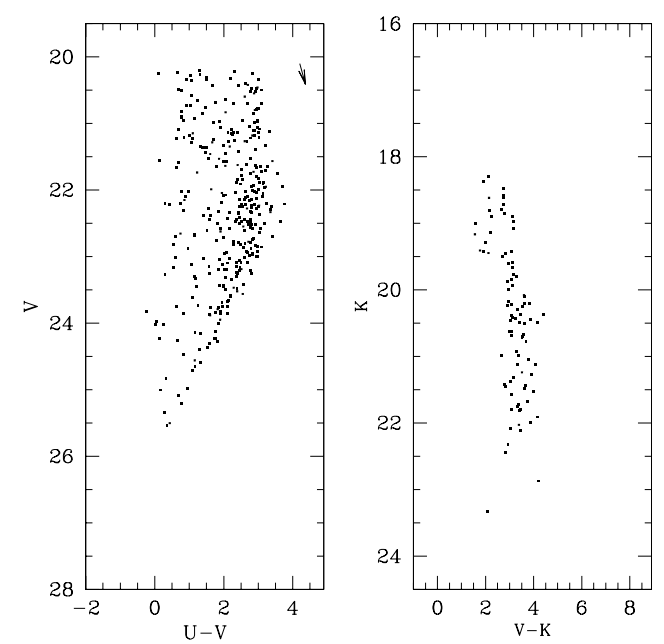

Fig. 7. $U V$ (left) and $V K$ (right) color-magnitude diagrams for foreground Galactic stars simulated using the Besançon group model. The correction for completeness and realistic photometric uncertainties have been applied by adding the simulated stars to our Field 2 images and re-measuring them. The reddening vector $E(B-V)=0.1$ is plotted in the upper right corner.

on the field of Soria et al. (1996), which was also observed in the near-IR (F110W and F160W filters) by Marleau et al. (2000), but the direct comparison is not possible due to different photometric bands used.

The only possible direct comparison is for our Field 1 photometry, which partially overlaps with the HST photometry of Mould et al. (2000) and the ground based photometry of Fasset \& Graham (2000). The last authors observed a wider field than ours in $U, B$ and $V$ Glass filters, but due to smaller telescope aperture $(2.5 \mathrm{~m})$, their photometry is much shallower. The HST photometry is deeper, but covers a much smaller area. The mean difference between $V$ magnitudes of Fasset \& Graham and Mould et al. photometry is $-0.13 \pm 0.07 \mathrm{mag}$, in the sense of HST photometry having a systematically fainter zero point (Fasset \& Graham 2000). Comparison of 26 stars in common between our data and that of Fasset \& Graham for the brightest blue stars (their Table 3 ) is presented in Fig. 8. The mean difference for all 26 stars is negligible for $V$-band photometry, but it amounts to $0.13 \mathrm{mag}$ in $U$-band. From Fig. 8 a systematic trend with the magnitude is apparent in $V$ and $U$ band. Excluding the star \#10 (see Table 2), which has HST $V$ magnitude of 21.04 (thus close to our value; star R4 in Table 1 of Mould et al.), and extended sources \#4, \#5 (see Rejkuba 2001; filled triangles in Fig. 8) and \#18, these trends can be represented by the following equations:

$$
\begin{aligned}
& V_{\mathrm{FORS} 1}-V_{\mathrm{FG}}=0.030 \times V_{\mathrm{FORS} 1}-0.65 \\
& U_{\mathrm{FORS} 1}-U_{\mathrm{FG}}=0.089 \times U_{\mathrm{FORS} 1}-2.05
\end{aligned}
$$

with rms equal to 0.055 for $V$ and 0.096 for $U$. At least part of this difference may be due to different filters used (Bessell 1995). Fasset \& Graham further neglected the 

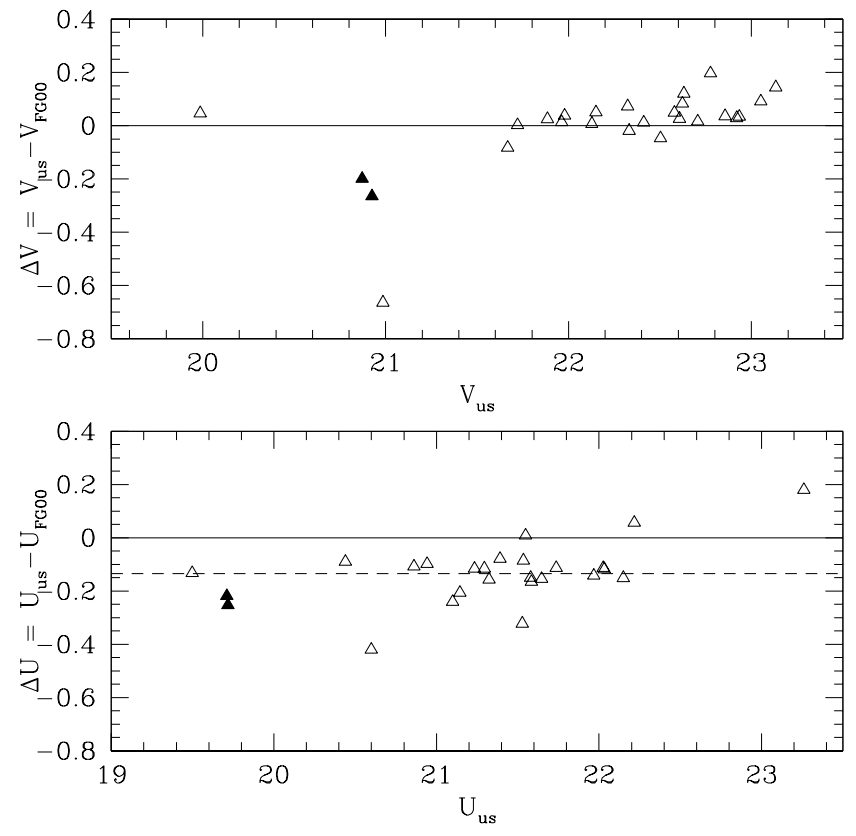

Fig. 8. Comparison of our photometry with that of Fasset \& Graham (2000) for 26 bright blue stars in common. In the upper panned the comparison of $V$ band magnitudes is presented and in the lower panel $U$-band magnitudes are compared. The two extended objects (\#4 and \#5 in Table 2) noted by Rejkuba (2001) are plotted with filled symbols. The mean offset of $-0.13 \mathrm{mag}$ in $U$-band is indicated with the dashed line.

color term in their calibration due to insufficient color coverage of their standards, while we found that the color term is important for our $U$-band calibration.

\section{The color-magnitude diagrams}

\subsection{Optical CMDs}

Optical CMDs probe young and intermediate age populations. Theoretically, the $U$ and $V$ light is dominated by young main sequence stars (Buzzoni 1995).

Figure 9 shows $(U-V)-V$ color-magnitude diagrams for stars in both observed halo fields of NGC 5128. Due to better seeing the saturation magnitude of the $V$-band is $\sim 0.3$ mag fainter for Field 2. Most of the stars redder than $(U-V) \sim 0$ belong to our own Galaxy (see Fig. 7). The most important characteristic of the $U V$ CMD of Field 1 is the upper main sequence, visible as the blue plume at $(U-V) \sim-1$ mag. By contrast, there are no such young massive stars in Field 2.

The Besançon Galaxy model (see previous section) was used to "clean" the CMDs of foreground star contamination. In Fig. 10 we show $U V$ CMDs after the subtraction of foreground stars. Overplotted are isochrones from Bertelli et al. (1994) for $Z=0.004$ and for $\log ($ age $)=7.0,7.3$, 7.5 for Field 1 and $\log ($ age $)=7.0,7.5$ and 7.8 for Field 2 . The isochrones were shifted to the distance of NGC 5128 , assuming a distance modulus of $(m-M)_{V}=27.8$ and reddening of $E(B-V)=0.11 \mathrm{mag}$ (Schlegel et al. 1998). The
Table 2. Comparison with Fasset \& Graham (FG) photometry; the numbers of stars in the first column are from FG.

\begin{tabular}{cccccc}
\hline \hline$\#$ & $V_{\mathrm{FG}}$ & $(B-V)_{\mathrm{FG}}$ & $(U-B)_{\mathrm{FG}}$ & $V_{\mathrm{FORS} 1}$ & $U_{\mathrm{FORS} 1}$ \\
\hline \hline 1 & 19.94 & +0.23 & -0.54 & 19.987 & 19.498 \\
$4^{a}$ & 21.07 & +0.12 & -1.26 & 20.871 & 19.711 \\
$5^{b}$ & 21.19 & +0.04 & -1.26 & 20.925 & 19.717 \\
$7^{c}$ & 21.51 & -0.07 & -0.34 & $\cdots$ & 20.291 \\
10 & 21.65 & +0.04 & -1.16 & 20.986 & 20.440 \\
12 & 21.72 & +0.21 & -0.20 & 21.722 & 21.580 \\
13 & 21.75 & +0.11 & -0.84 & 21.667 & 20.600 \\
15 & 21.86 & +0.09 & -0.61 & 21.885 & 21.099 \\
16 & 21.94 & -0.05 & -0.92 & 21.978 & 20.862 \\
17 & 21.95 & +0.34 & -0.82 & 21.963 & 21.391 \\
$18^{d}$ & 22.01 & +0.30 & -0.84 & $\ldots$ & $\cdots$ \\
20 & 22.10 & +0.07 & -0.82 & 22.150 & 21.144 \\
21 & 22.12 & -0.08 & -1.00 & 22.127 & 20.942 \\
26 & 22.25 & +0.08 & -0.98 & 22.323 & 21.234 \\
27 & 22.35 & -0.03 & -0.91 & 22.331 & 21.295 \\
28 & 22.40 & -0.05 & -0.87 & 22.412 & 21.323 \\
30 & 22.51 & +0.20 & -0.56 & 22.631 & 22.032 \\
32 & 22.53 & +0.13 & -0.50 & 22.579 & 22.217 \\
33 & 22.54 & +0.07 & -0.76 & 22.623 & 21.528 \\
35 & 22.55 & +0.11 & -0.55 & 22.503 & 21.968 \\
36 & 22.58 & -0.08 & -0.88 & 22.606 & 21.535 \\
37 & 22.58 & +0.02 & -1.06 & 22.777 & 21.549 \\
39 & 22.69 & -0.12 & -0.82 & 22.706 & 21.585 \\
42 & 22.82 & +0.00 & -1.02 & 22.856 & 21.646 \\
46 & 22.89 & +0.31 & -0.12 & 22.919 & 23.260 \\
47 & 22.90 & -0.05 & -1.00 & 22.934 & 21.737 \\
48 & 22.96 & +0.08 & -0.74 & 23.051 & 22.149 \\
50 & 22.99 & -0.04 & -0.81 & 23.134 & 22.027 \\
\hline & & & & &
\end{tabular}

${ }^{a}$ f1.GC-8 (Rejkuba 2001).

${ }^{b}$ f1.GC-25 (Rejkuba 2001).

${ }^{c}$ Saturated in FORS1 $V$ image.

${ }^{d}$ Extended in FORS1 images.

difference between the two diagrams is striking: the blue main sequence containing stars as young as $\sim 10$ Myr that is present in Field 1 is completely absent from the CMD of Field 2. Well separated from the main sequence in Field 1 is the sequence of blue core-helium burning (BHeB) stars at $(U-V) \sim 0.5$. The width of the gap and the tightness of the main sequence indicate low differential extinction in the field. In Field 2, the stars with $0.5<(U-V)<2.8$ and brighter than $V \lesssim 22.5$ have no corresponding main sequence stars and thus cannot be young stars migrating from blue to red during their core-He burning phase, as is the case for most of the objects in Field 1 with colors $0.5<(U-V)<2.5$. These stars in Field 2 are most probably the remaining foreground contamination, indicating that the foreground contamination may affect the numbers of blue and red HeB stars in Field 1 (see next paragraph). Only a few stars lie along the isochrone of $\log ($ age $)=7.5$ (Fig. 10), while most of them are consistent with much older ages. We conclude that there are no stars younger than $\sim 40$ Myr in Field 2 .

Note that isochrones for metallicities higher than $Z=$ 0.004 extend on the red supergiant edge to redder values 

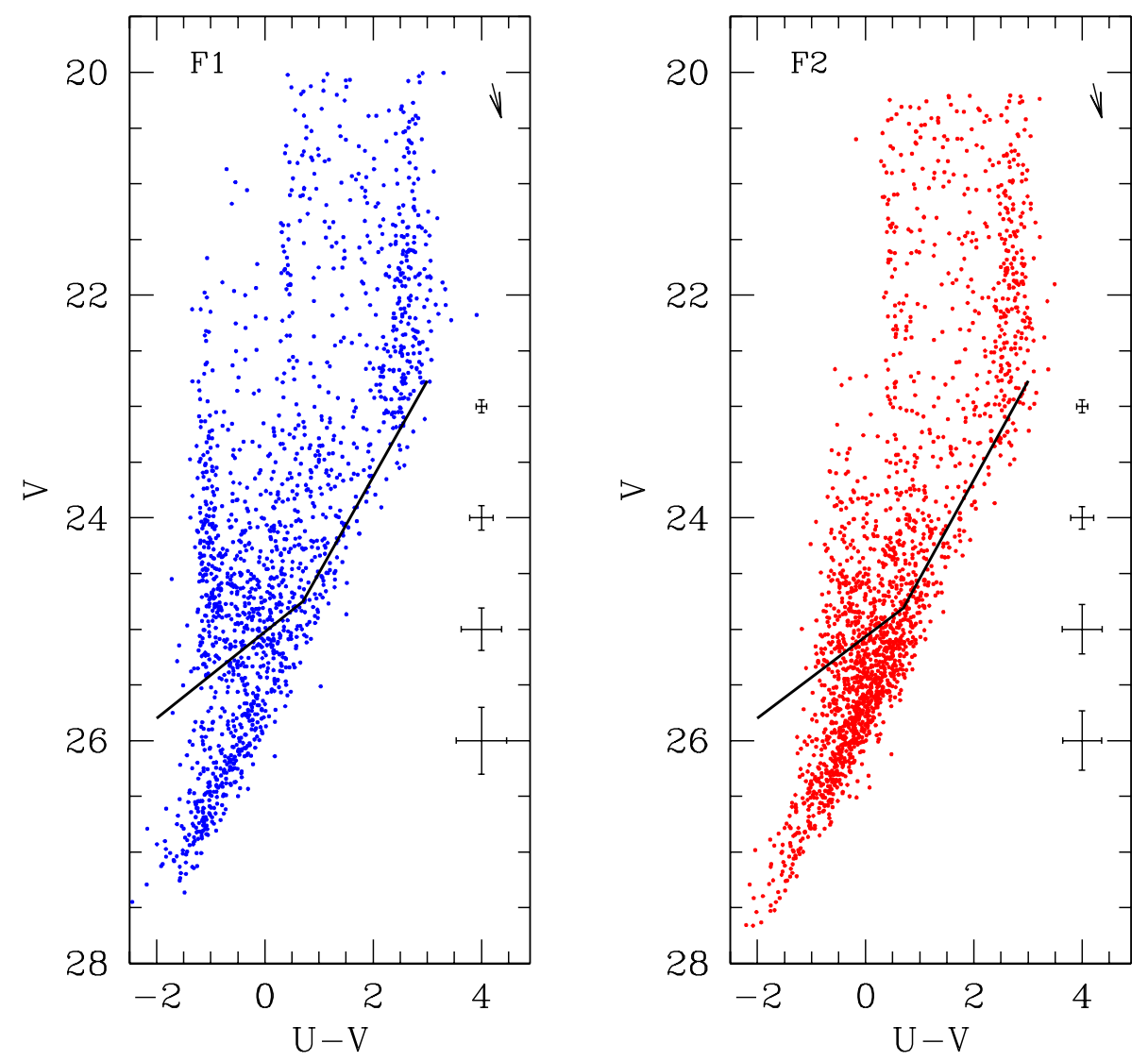

Fig. 9. UV color-magnitude diagram for Field 1 (left) and Field 2 (right). The arrow represents the reddening vector of $E(B-V)=0.1$. Typical photometry uncertainties are plotted on the right side. The solid line indicates the $50 \%$ completeness.

of $U-V$ than the reddest stars in Field 1 and thus do not fit well our observations. The ratio of blue to red supergiants strongly depends on metallicity (Langer \& Maeder 1995; Maeder \& Meynet 2001) and in principle could be used to constrain the metallicity of the youngest population in NGC 5128. Counting the number of blue and red supergiants for stars more massive than $\sim 12 M_{\odot}$, we find their ratio to be $B / R<0.7-0.8$ (although with high uncertainty due to the possible foreground contamination), in good agreement with the observed $B / R$ value in the SMC cluster NGC 300 (see discussion by Langer \& Maeder 1995). The metallicity of $Z=0.004$ (corresponding to $[\mathrm{Fe} / \mathrm{H}]=-0.7$ dex $)$ is appropriate for the SMC. However, since the $B / R$ value depends also on other parameters such as stellar mass, rotation and degree of overshooting (Maeder \& Meynet 2001), a more detailed comparison with models and the determination of the metallicity of this youngest stellar population in NGC 5128 is warranted (Rejkuba et al., in preparation).

The low metallicity implied by the fit of the isochrones on $U V$ CMDs probably reflects the metallicity of the gas left in the halo of NGC 5128 by the accreted satellite. Atomic HI (Schiminovich et al. 1994) and molecular CO gas (Charmandaris et al. 2000) present in Field 1 are slightly offset from the position of the diffuse stellar shell, the obvious remnant from the accreted galaxy.

\subsection{Optical-near IR CMDs}

Optical-near IR CMDs probe old and intermediate-age stellar populations. Theoretically, more than two thirds of the light in $K$-band is dominated by cool stars on the red giant branch (RGB) and asymptotic giant branch (AGB), and by red dwarfs (Buzzoni 1995). The red dwarfs are too faint to be detected at the distance of NGC 5128, and thus our $V K$ CMDs are entirely dominated by RGB and AGB stars (Fig. 11).

In Fig. 12 we show $V K$ CMDs of Fields 1 and 2 after the statistical subtraction of foreground stars. Overlaid are fiducial RGB sequences of Galactic globular clusters (from left to right: M15, 47 Tuc, NGC 6553 and NGC 6528; Ferraro et al. 2000) spanning a large range of metallicities $(-2.17 \leq[\mathrm{Fe} / \mathrm{H}] \leq-0.23 \mathrm{dex})$. As before, we used a distance modulus of 27.8 and reddening corresponding to $E(B-V)=0.1(E(V-K)=0.274$ and $A_{K}=0.0347$; Rieke \& Lebofsky 1985) to adjust the magnitudes and colors of RGB fiducials to those of NGC 5128. Obviously, most of the stars in Fig. 12 belong to the RGB. The right edge of the RGB is quite sharp, with most of the stars being more metal-poor than 47 Tuc $([\mathrm{Fe} / \mathrm{H}]=-0.71 \mathrm{dex})$ and none appearing to be as metalrich as NGC $6553([\mathrm{Fe} / \mathrm{H}]=-0.29 \mathrm{dex})$. However, the latter is due to incompleteness in $V$-band photometry. 

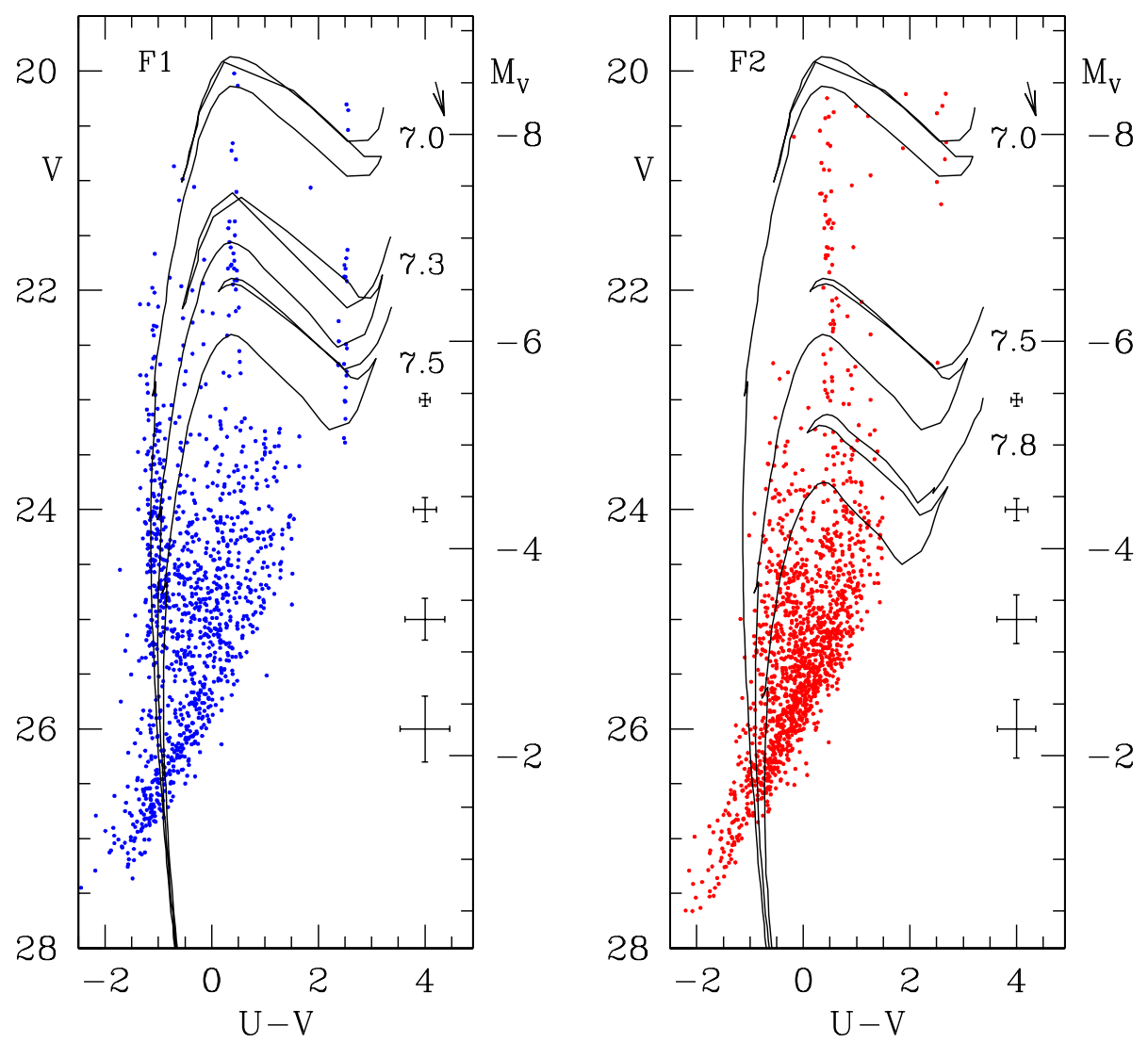

Fig. 10. CMDs with foreground stars statistically subtracted. Overplotted are isochrones from Bertelli et al. (1994) for $Z=0.004$ with $\log ($ age $)$ indicated with the number on the right side. The size of the reddening vector corresponds to $E(B-V)=0.1$ mag.

The spread in color of the RGB is larger than the photometric uncertainties (Fig. 13), indicating the presence of spread in metallicity and/or age. The most metal-poor stars have metallicities of -2 dex if their ages correspond to those of Galactic globular clusters. The population we probe with $V K$ photometry is more metal-poor than -0.7 dex. Our $V$-band images are not deep enough to detect more metal rich giants. Walsh et al. (1999) measured the mean oxygen abundance of five planetary nebulae in NGC 5128 to be $[\mathrm{O} / \mathrm{H}]=-0.5 \pm 0.3 \mathrm{dex}$, consistent with the presence of the large population of stars with metallicities below solar, as we observe in the $V K$ CMDs.

There are 1830 stars in Field 1 and 1197 in Field 2 which have good $K$-band photometry $\left(\sigma_{K}<0.5,-0.7<\right.$ sharp $<0.7$ and $\chi<2.0)$ above the respective $K$-band completeness limits, but which have not been detected in optical bands. They are uniformly distributed over all the ISAAC images. The large number of very red stars with good photometry in $K_{\mathrm{s}}$ and no counterpart in the $V$-band suggests that stars more metal-rich than $[\mathrm{Fe} / \mathrm{H}]=$ -0.7 dex are present, as expected for a luminous giant elliptical galaxy. This is in good agreement with the results of Harris et al. (1999) and Harris \& Harris (2000).

\section{The color-color diagram}

Figure 14 shows the color-color diagram for the objects detected in $U_{-}, V$ - and $K$-band in Field 1 (open squares) and Field 2 (filled triangles) that had ALLFRAME photometry uncertainties $<0.5$ mag and magnitudes brighter than the $50 \%$ completeness limits in all bands. The errorbars plotted are the ones given by ALLFRAME. A total of 99 objects in Field 1 and 90 in Field 2 satisfy these criteria. Most of them are located along the stellar sequence that crosses the color-color diagram in diagonal. Matching the $U V$ and $V K$ catalogues after the statistical subtraction of the foreground contamination, the stellar sequence disappears and only 54 and 44 objects with good photometry in all three filters are left in Field 1 and 2, respectively.

We use the color-color diagram presented in Fig. 14 to measure the reddening and the contamination by background galaxies.

\subsection{Reddening}

The objects crossing the color-color diagram in diagonal from top right to bottom left belong to the Milky Way. The quality of the fit of the stellar sequence with isochrones depends on the age of the isochrone with respect to stars. Because the foreground Milky Way stars span a range of ages, isochrones of different ages fit different parts of the stellar sequence. As an example, in order to guide the eye we overplotted the stellar loci of solar metallicity stars and $\log ($ age $)=7.0 \mathrm{yr}$ (Bertelli et al. 1994). The tight stellar sequence in the $U-V$ vs. $V-K$ 

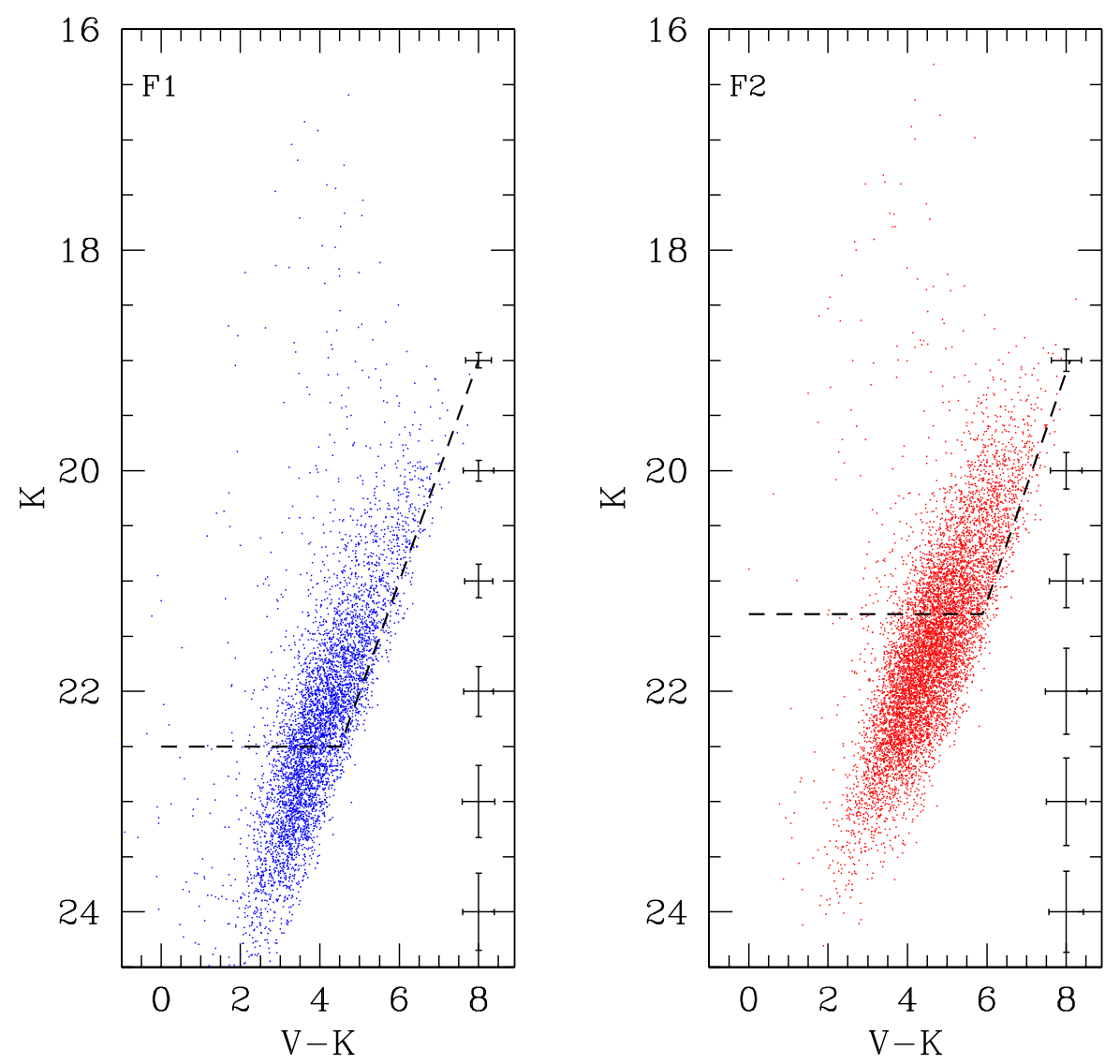

Fig. 11. $V K$ color-magnitude diagram for Field 1 (left) and Field 2 (right). The dashed line identifies the 50\% completeness level.

color-color diagram shows that the foreground reddening in the two fields is the same. Its magnitude was measured by fitting the theoretical isochrones and it amounts to $E(B-V)=0.15 \pm 0.05$ mag. This is in excellent agreement with the Schlegel et al. (1998) extinction maps and the reddening determined by Fasset \& Graham (2000), who used $U B V$ color-color diagrams to measure a value of $E(B-V)=0.14 \pm 0.02$ mag.

\subsection{Star-galaxy separation}

Using the color-color plots it is possible to distinguish the foreground dwarf stars from the most compact unresolved galaxies. Galaxies are located in the lower right part of the diagram in Fig. 14. The resolved galaxies are not plotted here since they got rejected by our selection of shape parameters in FIND and ALLFRAME. Note that the group of objects with $-0.5<(V-K)<2$ are the young stars detected in Field 1.

\section{Jet aligned star formation in the halo}

The chains of bright blue compact objects and optical filaments, first noticed by Blanco et al. (1975), were recognized as young blue supergiants and OB associations by Graham \& Price (1981) and Graham (1998). Our Field 1, situated $\sim 14 \mathrm{kpc}$ away from the center of the galaxy and coinciding with the NE radio lobe, contains part of this "string of blue knots and filaments."

In Fig. 15 we show the spatial distribution of the bluest (left) and the reddest (right) stars in both our fields. The red stars, belonging to the foreground Galactic population, are uniformly distributed in both fields. Field 2 has almost no very young blue stars, while the majority of the young population in Field 1 is aligned with the jet direction coming from the nucleus of the galaxy. The vertical structure to the right of the jet is located at the leftmost edge of a large Hi cloud found by Schiminovich et al. (1994). In the northern part of this field, a CO molecular cloud was recently discovered by Charmandaris et al. (2000).

Mould et al. (2000), Graham (1998) and Fasset \& Graham (2000) report the presence of collimated star formation in the halo of NGC 5128. They favor the scenario in which a past interaction between the radio jet (Morganti et al. 1999) and the Hi cloud complex (Schiminovich et al. 1994) is responsible for the star formation.

Finding active star formation far out in the halo of an elliptical galaxy is unusual. Understanding the triggering mechanism and the origin of the star formation, as well as the nature of the underlying ionized gas (Graham 1998; Morganti et al. 1991, 1992), is important for explaining the filamentary emission and jet-aligned structures observed 

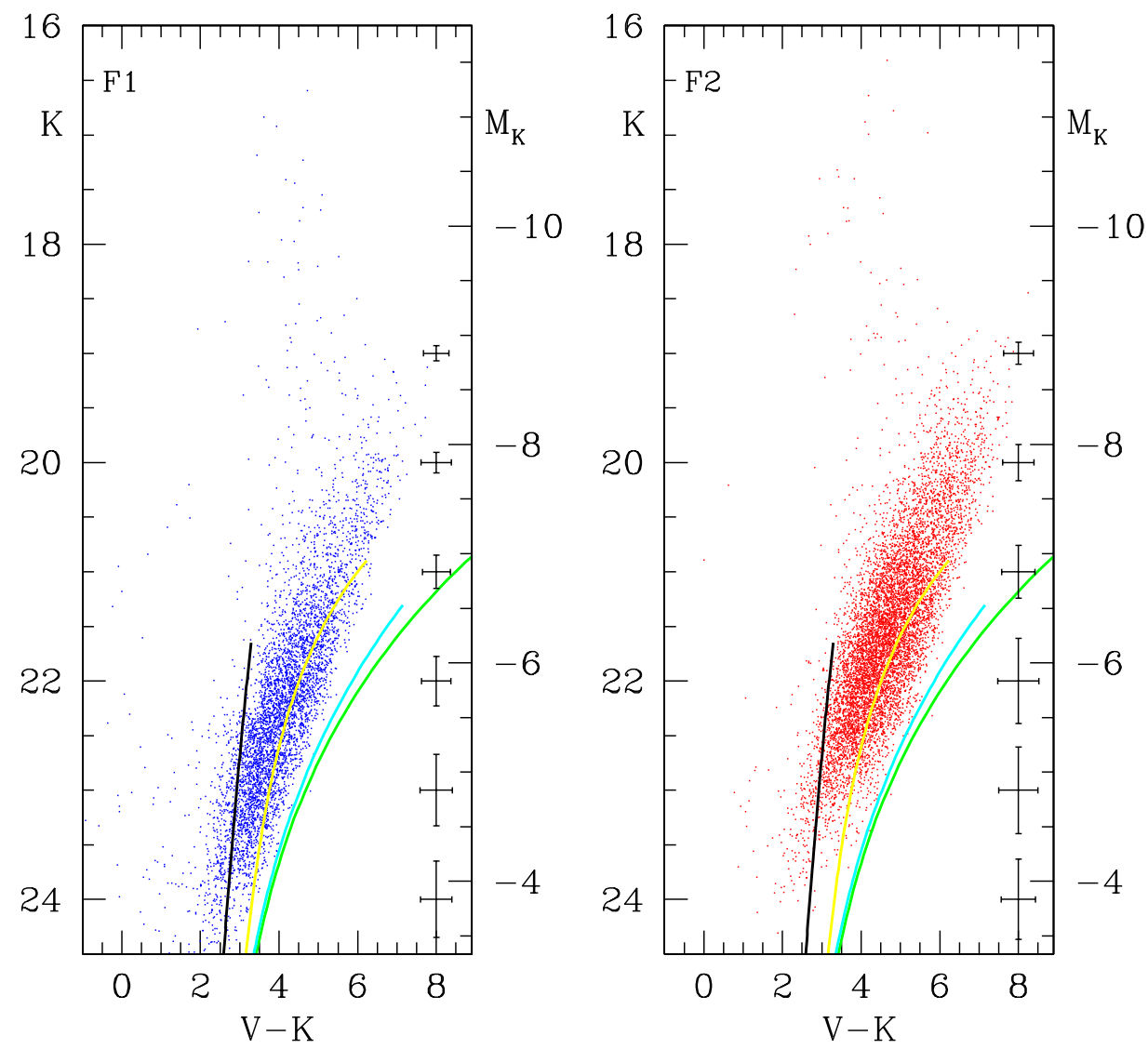

Fig. 12. $V K$ CMDs with foreground stars statistically subtracted. Overplotted are fiducial RGB sequences of Galactic globular clusters (from left to right): M 15, 47 Tuc, NGC 6553 and NGC 6528, from Ferraro et al. (2000).

in other galaxies (e.g., in NGC 1275: Lazareff et al. 1989; Sabra et al. 2000; in M87: Gavazzi et al. 2000; and in other more distant radio sources: Rees 1989, Best et al. 1996, De Breuck et al. 1999).

The alignment of the luminous blue stars with the radio axis of NGC 5128 (Fig. 15) may shed some light on the debated issue of the observed alignment of the radio and optical structures in high- $z$ radio galaxies (Rees 1989; Best et al. 1996). One of the proposed alignment models is based on the assumption that the action of the jets tunneling through the galactic medium affects the gas via shock heating, thereby inducing vigorous star formation (Begelman \& Cioffi 1989; Rees 1989; Daly 1990). In particular, as Rees (1989) discussed in detail, the stars form from the cool-phase gas (with $T \leq 10^{4} \mathrm{~K}$ ) in clouds massive or dense enough to be Jeans-unstable.

This mechanism may work well for massive high- $z$ radio galaxies, in which most of the baryonic mass would still be gaseous. In the present-day giant ellipticals, on the other hand, usually little gas is left to support strong star formation. However, probably due to previous accretion of a gas-rich galaxy, NGC 5128 has substantial cool, dense clouds of molecular CO and atomic Hi gas in its halo (Schiminovich et al. 1994; Charmandaris et al. 2000). We note that these clouds could form the clumpy ISM/IGM necessary for the "bursting bubble" model proposed by Morganti et al. (1999).
We have shown clear evidence for blue stars as young as $10 \times 10^{6}$ yr (Fig. 10). We also see underlying emission, particularly in our $U$-band images, which probably comes from the [OII] 3727 emission line. High-resolution spectra with good signal-to-noise ratios are needed to decide whether this emission is due to gas being photoionized by the newly formed stars or being photoionized by particles coming directly from the nucleus.

Other low-redshift galaxies also show filamentary ionized gas structures in their halos. In the case of M 87 (Gavazzi et al. 2000), the filament of ionized gas in the NE part of the halo coincides with the Eastern radio-lobe, in much the same way as in the much closer NGC 5128. It would be interesting to search for young stars associated with that filament too.

\section{Intermediate age population}

In contrast with the conclusions of HST studies (Harris et al. 1999; Harris \& Harris 2000) of stellar populations in NGC 5128, we detect not only old population II stars, but also a significant number of stars with magnitudes brighter than the tip of $\operatorname{RGB}\left(M_{K}^{\mathrm{Tip}}=-0.64( \pm 0.12)[\mathrm{M} / \mathrm{H}]-\right.$ $6.93( \pm 0.14)$; Ferraro et al. 2000).

We took bolometric corrections from Bessell \& Wood (1984) and the empirical fit of $(V-K)$ vs. $T_{\text {eff }}$ from Bessell et al. (1998) to transform our $K-(V-K)$ CMDs to the 

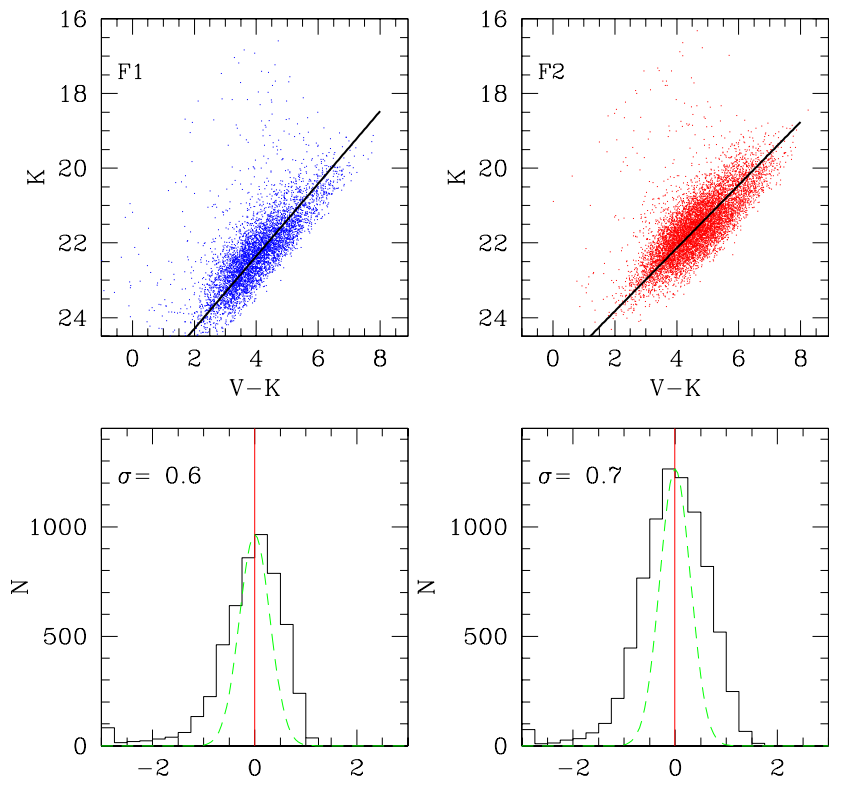

Fig. 13. The histogram of the observed color distribution along the RGB (lower panels) is plotted accounting for the actual slope of the RGB (thick line in upper panels) in Field 1 (left) and 2 (right). The $1 \sigma$ spread in color of the RGB is indicated in the upper left corner. Overplotted with the dashed line is a Gaussian with the $\sigma=0.3$, typical of the distribution from the photometry errors.

theoretical plane (Fig. 16). Overplotted on the H-R diagrams are the Padova tracks from Girardi et al. (2000) for the first-ascent giant branch stars with masses $M=0.6$, $0.8,1.2$ and $1.6 M_{\odot}$ and metallicities $Z=0.004$ (full lines) and $Z=0.008$ (dashed lines). The sharp cut-off on the right side of the $\mathrm{H}-\mathrm{R}$ diagrams is due to incompleteness in $V$-band.

In discussing the H-R diagram, we should consider blending. According to theoretical predictions (Renzini 1998), at the fiducial galactocentric distances of $9,12.8$ and $17.9 \mathrm{kpc}$, the number of blends consisting of 2 stars belonging to the tip of RGB is $\sim 1300,300$ and 55 stars (per $2.2^{\prime} \times 2.2^{\prime}$; i.e. field of view of ISAAC), respectively. In this calculation, the $B$-band surface brightness measurements from Mathieu et al. (1996) were used. Obviously, the innermost regions of the galaxy, at the distance $<10 \mathrm{kpc}$ from the nucleus are too crowded to give accurate photometry for RGB stars and most of the stars above the tip of RGB.

We attempted to use the NIC3 HST images overlapping with our Field 2 (galactocentric distance $R_{\mathrm{gc}} \sim$ $9 \mathrm{kpc}$ ) data to improve the resolution. However, we found out that many faint stars visible on ISAAC images are completely within the noise of NIC3 data. The resolution of our ISAAC data in the best seeing is as good as that of NICMOS images, with the advantage of having much better $\mathrm{S} / \mathrm{N}$ and a more stable PSF. The confirmation of the number of AGB stars in Field 2 can be obtained only through the analysis of the LPVs (Rejkuba et al. in preparation).

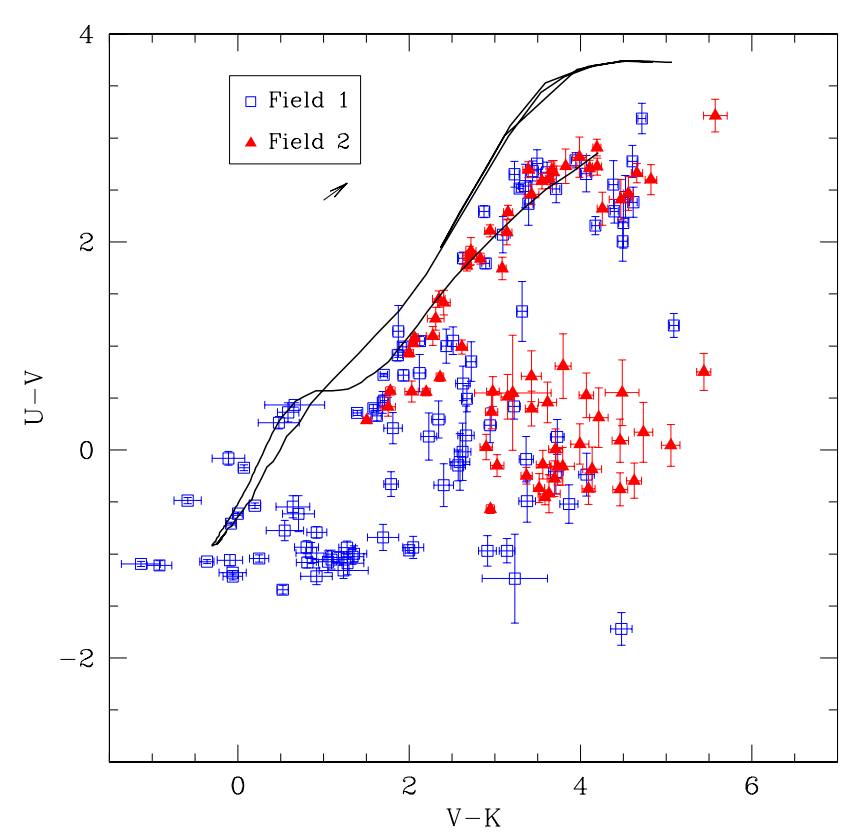

Fig. 14. Color-color diagram for the objects matched in $U, V$ and $K_{\mathrm{s}}$ frames that had ALLFRAME photometry uncertainties $<0.5$ mag and magnitudes brighter than the $50 \%$ completeness limits in all bands. Field 1 objects are plotted with open squares and Field 2 with filled triangles. Overplotted is the Padova isochrone (Bertelli et al. 1994) for the solar metallicity and $\log ($ age $)=7.0 \mathrm{yr}$ that indicates the locus of the foreground stellar sequence. The reddening vector has dimensions corresponding to $E(B-V)=0.1$.
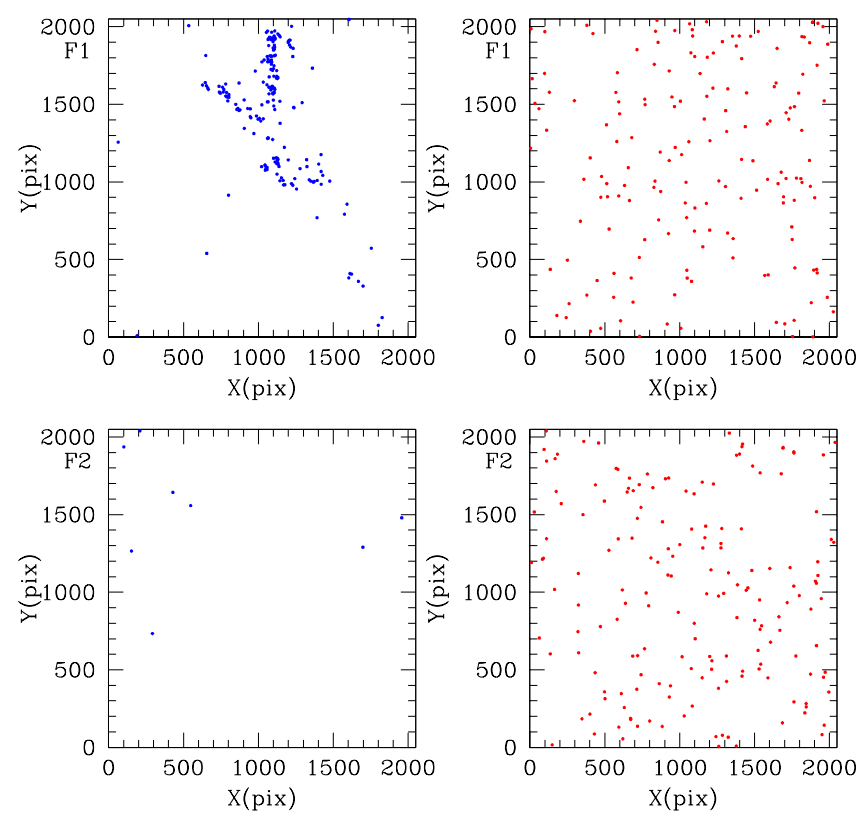

Fig. 15. The spatial distribution of the bluest $(U-V)<-0.75$ (left panels) and the reddest $(U-V)>2$ (right panels) stars in Field 1 (top panels) and 2 (bottom panels) for stars with magnitudes above the $50 \%$ completeness limit $(V<25$ and $U<25)$. The north is up and east is to the left.

In Field 1 ( $R_{\mathrm{gc}} \sim 14 \mathrm{kpc}$ ), our outer shell field, crowding is not that severe. The expected number of two-star 

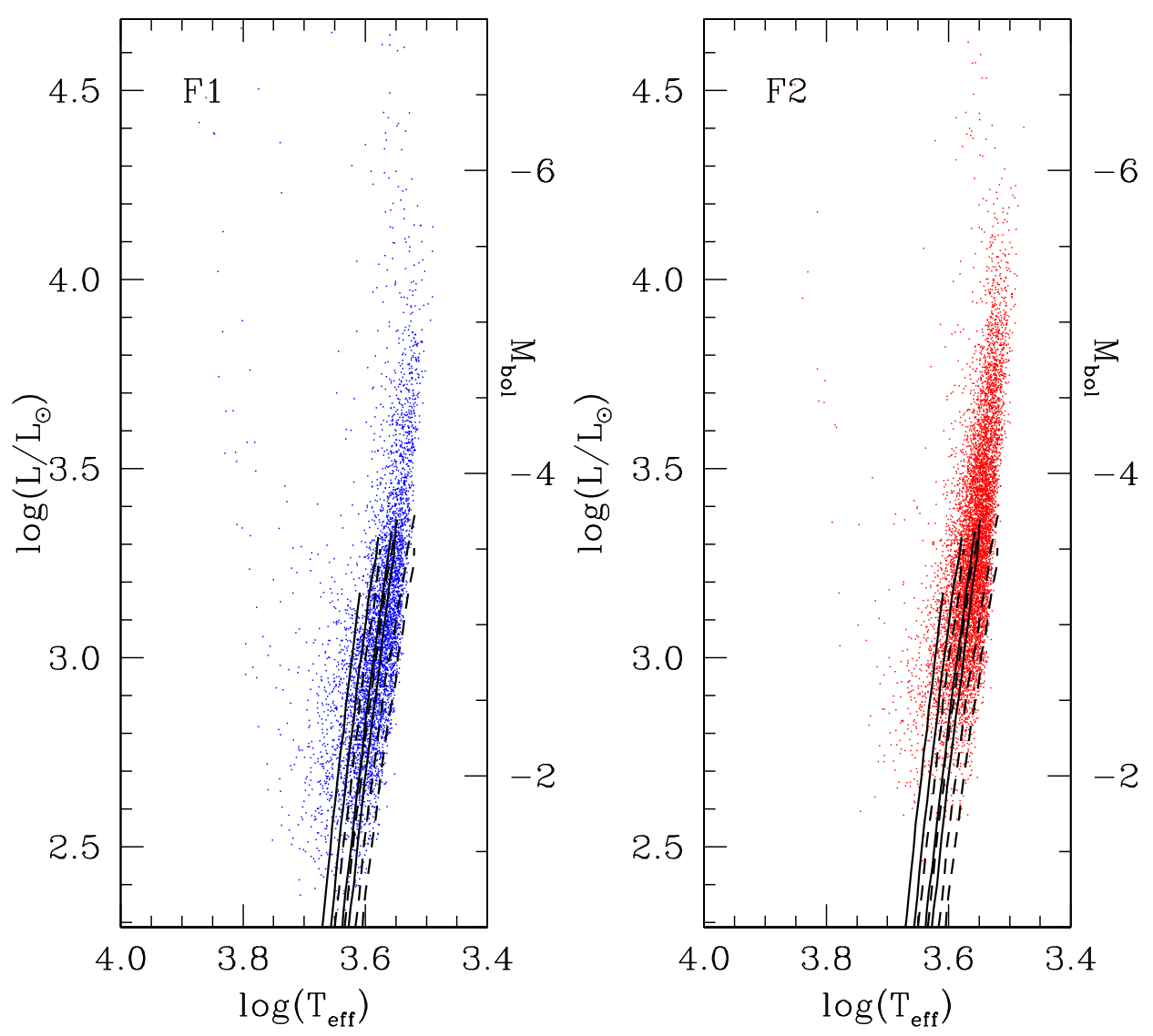

Fig. 16. The HR diagram for old and intermediate stellar populations in Field 1 and 2. Overplotted are evolutionary tracks from Padova (Girardi et al. 2000) for stars with masses $M=0.6,0.8,1.2$ and $1.6 M_{\odot}$ and $Z=0.004$ (full line) and $Z=0.008$ (dashed line).

blends at the RGB tip in this field ranges from 300-50, as the surface brightness drops across the field. The total number of such blends is therefore less than $\sim 200$ in the most pessimistic calculation. On the other hand, the number of stars above the tip of RGB $\left(M_{\mathrm{bol}} \sim-3.7\right.$; Girardi et al. 2000) is 768. Subtracting the number of possible blends and allowing for a few of the brightest and bluest stars $\left(\sim 30\right.$ stars with $\left.M_{K}<9\right)$ to be the remaining foreground contamination, there are still more than 500 stars whose position in the $\mathrm{H}-\mathrm{R}$ diagram and CMD is consistent with an intermediate-age AGB population.

In the inner halo field, Field 2, the number of stars above the tip of RGB is 2844 . After subtracting the number of expected blends $(\lesssim 1500)$, the number of AGB stars is 2.5 times larger than in Field 1. This confirms the presence of gradients in the intermediate-age population within the halo of NGC 5128, as suggested by Marleau et al. (2000).

The intermediate-age AGB population could have easily been missed in $V$ and $I$-band HST studies due to small field of view and small ( $<0.5 \mathrm{mag})$ optical magnitude difference between the tip of the RGB and the tip of AGB. The AGB stars are up to $\sim 2$ mag brighter in $M_{K}$ and $M_{\text {bol }}$ than RGB stars and thus are easily detectable in near-IR. Thanks to this, Marleau et al. (2000) could detect some AGB stars in the much smaller NICMOS field.
The brightest stars in M 32, in the Galactic bulge and the bulge of M 31 have similar brightnesses, reaching $M_{\text {bol }}=-5.5$ (Freedman 1992; Elston \& Silva 1992; Frogel \& Whitford 1987; Rich \& Mould 1991). Due to this similarity, Davidge \& van den Bergh (2001) suggested that the tip of AGB could be used as a standard candle for determination of distances. In Fig. 17 we present the $K_{\mathrm{s}}$-band and bolometric magnitude luminosity functions. The tip of the AGB is observed at a bolometric magnitude of -5 in both fields (in spite of the crowding in Field 2), consistent with the adopted distance modulus for NGC 5128. Most of the stars brighter than this magnitude (as well as stars brighter than $M_{K}=-9$; see left panels in Fig. 17) probably belong to our Galaxy.

\section{The shell}

In our deep $V$-band image of Field 1, the prominent NE shell is easily recognizable as the region with higher surface brightness, and also with higher density of stellar objects. All the stars that are spatially coincident with the shell on the $V$-band image are shown in Fig. 18. In the left two panels we plot the $U V$ CMD of all stars coincident with the position of the diffuse shell (middle panel), while the corresponding $V K \mathrm{CMD}$ is on the right. The much smaller field of view of ISAAC is centered on the shell so 

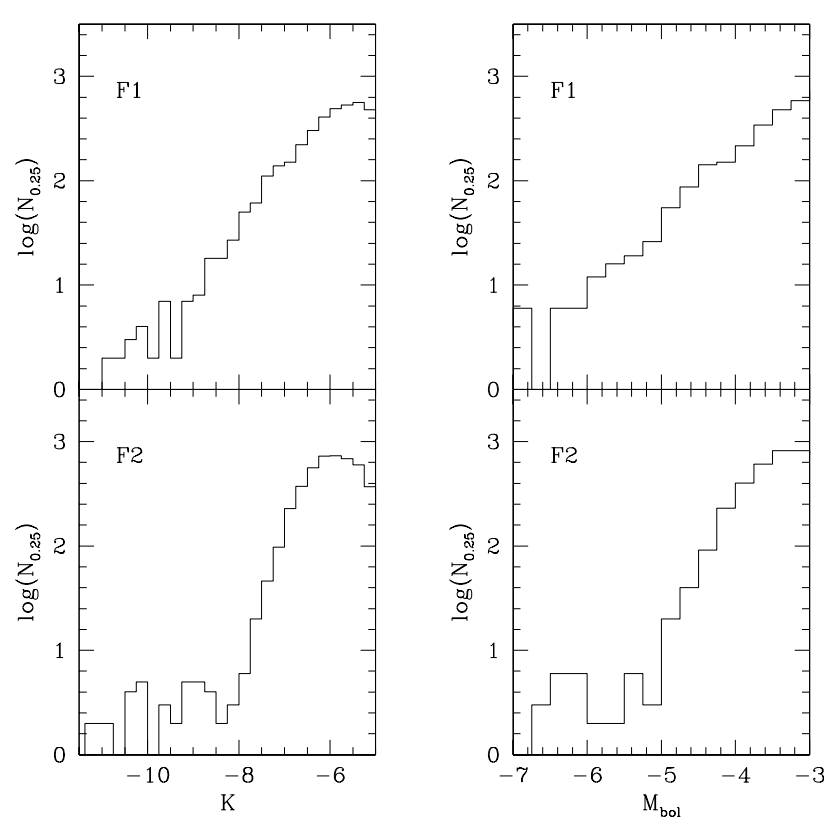

Fig. 17. $K$-band (left) and bolometric magnitude luminosity functions (right) for stars redder than $\left(V-K_{\mathrm{s}}\right)>2$ in Fields 1 and 2 .

that the shell stars cover most of the image. Note that projected on the shell is the stream of blue, young objects that are aligned with the jet direction. Excluding the stars that are coincident with the star-forming area (lower panels), the stellar population belonging to the shell is very similar to the one observed in the halo of NGC 5128, i.e., our Field 2 population (Figs. 9, 11). As in the rest of the galaxy there is a significant spread in metallicity.

The presence of stellar shells or ripples around earlytype galaxies is usually explained as evidence of the accretion and later merging of a smaller companion galaxy. The simulations of the stellar component in the merging galaxies have shown that the shells are created as a result of phase-wrapping or of spatial-wrapping of the tidal debris (Quinn 1984; Hernquist \& Quinn 1989). The companion galaxies may contain different stellar populations with respect to the giant elliptical (e.g. most dwarf galaxies have much lower metallicity than giants Mateo 1998).

Here for the first time we obtained the magnitudes and colors of stars belonging to a shell. They are surprisingly similar to the rest of the halo stars in NGC 5128. However, due to incompleteness of our $V$-band photometry, we cannot probe the most metal-rich part of the halo of NGC 5128. Very high resolution and deeper observations of the shell stars are possible with the Advanced Camera for Surveys at Hubble Space Telescope. In order to be able to better separate the stars that genuinely belong to shells from the "normal" halo stars in NGC 5128 and confirm the present result a more quantitative comparison between the stellar populations in our two fields is necessary (Rejkuba et al., in preparation).

\section{Summary}

Using the VLT with FORS1 and ISAAC, we have resolved stars in the halo and in the diffuse north-eastern shell of the closest giant elliptical galaxy, NGC 5128. With the $U, V$ and $K_{\mathrm{s}}$-band photometry we probe different stellar populations. The $U$ filter, in particular, is sensitive to the youngest stars. It has revealed the striking difference in the star formation history between the the inner halo field (Field 2) and outer halo field (Field 1), the latter coinciding with the diffuse shell that is presumably the signature of a recent merger.

In Field 1, stars as young as 10 Myr are present, while there are no stars younger than at least 40 Myr in Field 2. Thanks to the high quality of our $U V$ photometry, we also detect the gap between the main sequence and the blue core-helium burning supergiants. The foreground and background contamination has been corrected using the Besançon Galaxy model and our own color-color diagram.

The recent star formation in Field 1 is approximately aligned with the direction of the jet coming from the center of the AGN. However, there is also a chain of young blue stars offset from the jet direction that is aligned with the edge of the molecular clouds. The presence of the atomic and molecular gas (Schiminovich et al. 1994; Charmandaris et al. 2000) and their association with the diffuse stellar shell is compatible with the dynamical scenario of phase-wrapping following the merger of a smaller gas-rich galaxy with NGC 5128 (Quinn 1984). In this scenario, the interaction of the material coming from the AGN in the center of the galaxy with the material left from the merger stimulated the star formation in the halo, $\sim 15 \mathrm{kpc}$ away from the galactic centre. The metallicity of the newly formed stars gives an upper limit on the abundance of the gas of the accreted satellite with the value of $Z=0.004$, a value typical of the SMC.

While the $U V$ CMDs probed the youngest stellar populations, the combination of optical and near-IR filters is most sensitive to old and intermediate-age populations. In our $V K$ CMDs we observe a very wide and extended giant branch. The width indicates the presence of Population II stars with metallicities ranging from -2 to -0.7 dex. Unfortunately, our $V$-band images are not deep enough to detect more metal-rich stars, but the large number of stars detected on $K$-band images without a counterpart in $V$-band suggests an even redder and more metal-rich RGB population, in agreement with the previous HST studies (Soria et al. 1996; Harris et al. 1999; Harris \& Harris 2000).

In contrast with the previous two studies, we find a very extended giant branch up to $M_{\text {bol }}=-5$, which reveals the presence of the intermediate-age AGB population. Our extensive crowding experiments and theoretical predictions of the amount of blending (Renzini 1998) demonstrate that blending due to crowding is not significant enough to mimic this population in the outer field (Field 1). In the inner field, the extent and the properties of the AGB population will be assessed through the study 

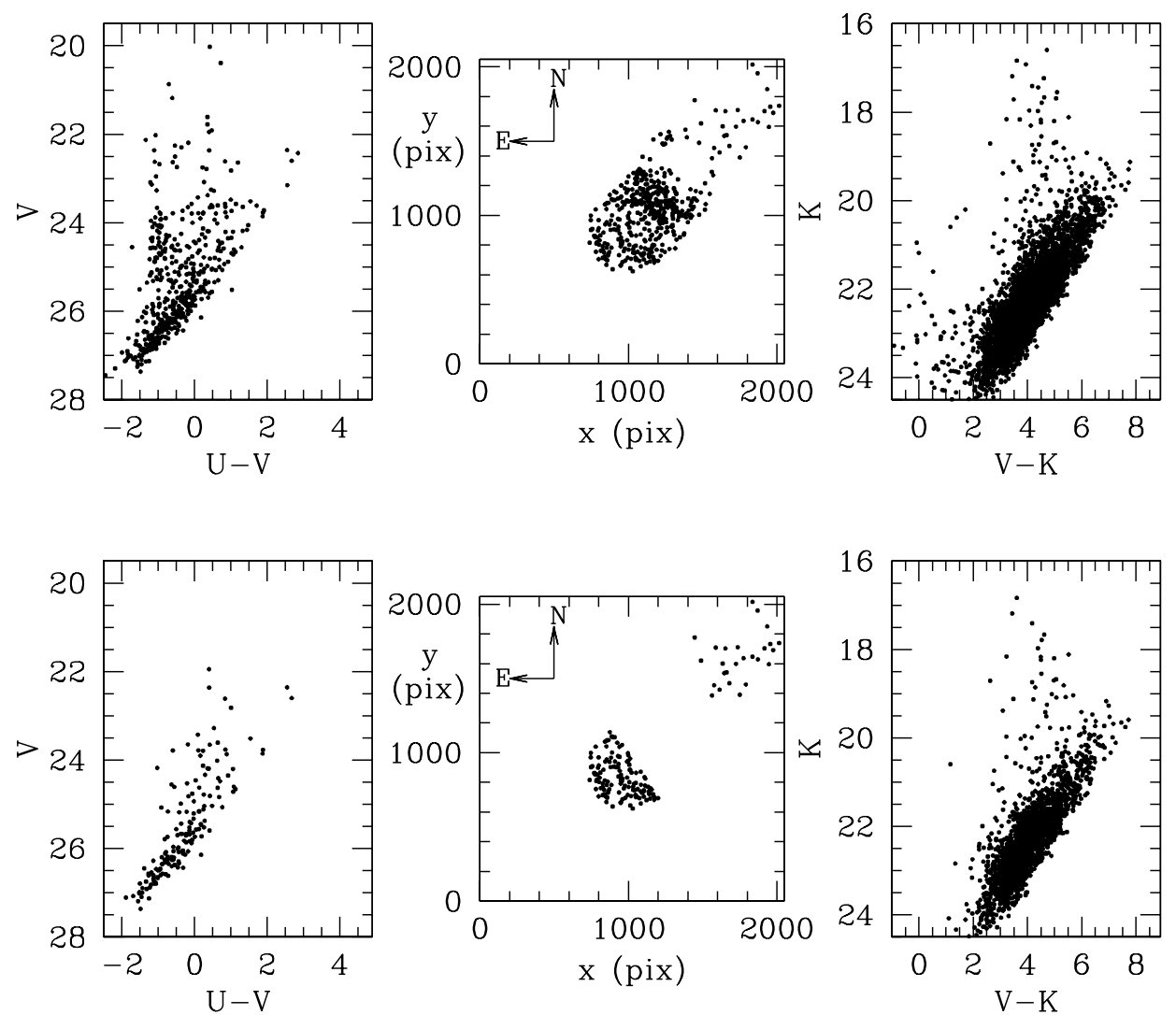

Fig. 18. The color-magnitude diagrams for the stars located at the position of the diffuse NE shell.

of the long period variables that have been detected in both fields (Rejkuba et al. in preparation).

Acknowledgements. We thank Manuela Zoccali for help with DAOPHOT and ALLFRAME and Joel Vernet for help with IDL. We thank Francesco Ferraro who kindly provided the tables of $V K$ globular cluster fiducial RGB sequences in electronic form. Felipe Barrientos kindly provided the $U-V$ vs. $V-K$ colors for galaxies of different morphological types and redshifts. MR acknowledges ESO studentship programme. TRB is grateful to the Australian Research Council and P. Universidad Católica for financial support and to the DITAC International Science \& Technology Program. This work was supported by NASA Grant GO-07874.01-96A and by the Chilean Fondecyt No. 01990440 and 799884. Finally, we are grateful to the referee Mario Mateo for his helpful and thorough report.

\section{References}

Aparicio, A. 1998, in IAU Symp. 192 - The Stellar Content of Local Group Galaxies, ed. P. Whitelock, \& R. Cannon, 20 Begelman, M. C., \& Cioffi, D. F. 1989, ApJ, 345, L21

Bertelli, G., Bressan, A., Chiosi, C., Fagotto, F., \& Nasi, E. 1994, A\&AS, 106, 275

Bessell, M. S. 1995, PASP, 107, 672

Bessell, M. S., \& Wood, P. R. 1984, PASP, 96, 247

Bessell, M. S., Castelli, F., \& Plez, B. 1998, A\&A, 333, 231

Best, P. N., Longair, M. S., \& Röttgering, H. J. A. 1996, MNRAS, 280, L9

Bica, E. 1988, A\&A, 195, 76
Blanco, V. M., Graham, J. A., Lasker, B. M., \& Osmer, P. S. 1975, ApJ, 198, L63

Bruzual, A. G., \& Charlot, S. 1993, ApJ, 405, 538

Buzzoni, A. 1995, ApJS, 98, 69

Charmandaris, V., Combes, F., \& van der Hulst, J. M. 2000, A\&A, 356, L1

Chiosi, C., Bertelli, G., \& Bressan, A. 1992, ARA\&A, 30, 235

Clarke, D. A., Burns, J. O., \& Norman, M. L. 1992, ApJ, 395, 444

Cooper, B. F. C., Price, R. M., \& Cole, D. J. 1965, Aust. J. Phys., 18, 589

Daly, R. A. 1990, ApJ, 355, 416

Davidge, T. J., \& van den Bergh, S. 2001, ApJ, 553, L133

De Breuck, C., van Breugel, W., Minniti, D., et al. 1999, A\&A, 352, L51

Dufour, R. J., \& van den Bergh, S. 1978, ApJ, 226, L73

Elston, R., \& Silva, D. R. 1992, AJ, 104, 1360

Fassett, C. I., \& Graham, J. A. 2000, ApJ, 538, 594

Feigelson, E. D., Schreier, E. J., Delvaille, J. P., et al. 1981, ApJ, 251, 31

Ferraro, F. R., Montegriffo, P., Origlia, L., \& Fusi Pecci, F. 2000, AJ, 119, 1282

Freedman, W. L. 1992, AJ, 104, 1349

Frogel, J. A., \& Whitford, A. E. 1987, ApJ, 320, 199

Gallart, C. 1998, ApJ, 495, L43

Gallart, C., Freedman, W. L., Mateo, M., et al. 1999, ApJ, 514, 665

Gavazzi, G., Boselli, A., Vílchez, J. M., Iglesias-Paramo, J., \& Bonfanti, C. 2000, A\&A, 361, 1

Girardi, L., Bressan, A., Bertelli, G., \& Chiosi, C. 2000, A\&AS, 141,1

Graham, J. A. 1979, ApJ, 232, 60 
Graham, J. A. 1998, ApJ, 502, 245

Graham, J. A., \& Price, R. M. ApJ, 247, 813

Grebel, E. K. 2000, in The Evolution of Galaxies. I.Observational Clues, ed. J. M. Vilchez, G. Stasinska, \& E. Perez (Kluwer, Dordrecht)

Harris, G. L. H., \& Harris, W. E. 2000, AJ, 120, 2423

Harris, G. L. H., Harris, W. E., \& Poole, G. B. 1999, AJ, 117, 855

Held, E. V., Saviane, I., Momany, Y., \& Carraro, G. 2000, ApJ, 530, L85

Hernquist, L., \& Quinn, P. S. 1989, ApJ, 342, 1

Hui, X., Holland, C. F., Freeman, K. C., \& Dopita, M. A. 1995, ApJ, 449, 592

Hurley-Keller, D., Mateo, M., \& Nemec, J. 1998, AJ, 115, 1840

Israel, F. P. 1998, A\&AR, 8, 237

Jerjen, H., \& Rejkuba, M. 2001, A\&A, 371, 487

Kennicutt, R. C. 1998, ARA\&A, 36, 189

Kraft, R. P., Forman, W., Jones, C., et al. 2000, ApJ, 531, L9

Kurucz, R. L. 1998, IAU Symp. 189, ed. T. R. Bedding, A. J. Booth, \& J. Davis (Kluwer, Dordrecht), 217

Landolt, A. U. 1992, AJ, 104, 340

Langer, N., \& Maeder, A. 1995, A\&A, 295, 685

Lazareff, B., Castets, A., Kim, D.-W., \& Jura, M. 1989, ApJ, 336, L13

Maeder, A., \& Meynet, G. 2001, A\&A, in press [astro-ph/0105051]

Malin, D. F., Quinn, P. J., \& Graham, J. A. 1983, ApJ, 272, L5

Maraston, C. 1998, MNRAS, 300, 872

Marleau, F. R., Graham, J. R., Liu, M. C., \& Charlot, S. 2000, AJ, 120,1779

Mateo, M. 1998, ARA\&A, 36, 435

Mathieu, A., Dejonghe, H., \& Hui, X. 1996, A\&A, 903, 30

Minniti, D., \& Zijlstra, A. A. 1997, AJ, 117, 1743

Minniti, D., \& Zijlstra, A. A. 1996, ApJ, 467, L13

Minniti, D., Alonso, M. V., Goudfrooij, P., Jablonka, P., \& Meylan, G. 1996, ApJ, 467, 221

Morganti, R., Killeen, N. E. B., Ekers, R. D., \& Oosterloo, T. A. 1999, MNRAS, 307, 750

Morganti, R., Fosbury, R. A. E., Hook, R. N., Robinson, A., \& Tsvetanov, Z. 1992, MNRAS, 256, 1

Morganti, R., Robinson, A., Fosbury, R. A. E., et al. 1991, MNRAS, 249, 91

Mould, J. R., Ridgewell, A., Gallagher, J. S. III, et al. 2000, ApJ, 536, 266
Persson, S. E., Murphy, D. C., Krzeminski, W., Roth, M., \& Rieke, M. J. 1998, AJ, 116, 2475

Quinn, P. J. 1984, ApJ, 279, 596

Rees, M. J. 1989, MNRAS, 239, 1

Rieke, M. J., \& Lebofsky, M. J. 1985, ApJ, 288, 618

Rejkuba, M. 2001, A\&A, 369, 812

Rejkuba, M., Minniti, D., Gregg, M. D., et al. 2000, AJ, 120, 801

Renzini, A. 1998, AJ, 115, 2459

Renzini, A., \& Fusi Pecci, F. 1988, ARA\&A, 26, 199

Rich, R. M., \& Mould, J. R. 1991, AJ, 101, 1268

Robin, A., \& Creze, M. 1986, A\&A, 157, 71

Robin, A. C., Haywood, M., Creze, M., Ojha, D. K., \& Bienayme, O. 1996, A\&A, 305, 125

Sabra, B. M., Shields, J. C., \& Filippenko, A. V. 2000, ApJ, 545,157

Saracco, P., Giallongo, E., Cristiani, S., et al. 2001, A\&A, in press [astro-ph/0104284]

Schlegel, D. J., Finkbeiner, D. P., \& Davis, M. 1998, ApJ, 500, 525

Schiminovich, D., van Gorkom, J. H., van der Hulst, J. M., \& Kasow, S. 1994, ApJ, 423, L101

Schreier, E. J., Burns, J. O., \& Feigelson, E. D. 1981, ApJ, 251, 523

Soria, R., Mould, J. R., Watson, A. M., et al. 1996, ApJ, 465, 79

Stanford, S. A., Eisenhardt, P. R. M., \& Dickinson, M. 1995, ApJ, 450, 512

Stetson, P. B. 1994, PASP, 106, 250

Stetson, P. B. 1987, PASP, 99, 191

Tolstoy, E. 1998, in Dwarf Galaxies \& Cosmology, ed. T. X. Thuan, C. Balkowski, V. Cayatte, \& J. Tran Thanh Van [astro-ph/9807154]

Tonry, J. L., \& Schechter, P. L. 1990, AJ, 100, 1794

Unger, S. J., Clegg, P. E., Stacey, G. J., et al. 2000, A\&A, 355, 885

van den Bergh, S. 2000, The Galaxies of the Local Group (Cambridge University Press)

van den Bergh, S. 1999, A\&ARv, 9, 273

Vazdekis, A., Salaris, M., Arimoto, N., \& Rose, J. A. 2001, ApJ, 549, in press

Walsh, J. R., Walton, N. A., Jacoby, G. H., \& Peletier, R. F. 1999, A\&A, 346, 753

Wild, W., \& Eckart, A. 2000, A\&A, 359, 483 